\title{
Convergent Rhythm Generation from Divergent Cellular Mechanisms
}

\author{
Jason C. Rodriguez, Dawn M. Blitz, and Michael P. Nusbaum \\ Department of Neuroscience, Perelman School of Medicine, University of Pennsylvania, Philadelphia, Pennsylvania 19104
}

Different modulatory inputs commonly elicit distinct rhythmic motor patterns from a central pattern generator (CPG), but they can instead elicit the same pattern. We are determining the rhythm-generating mechanisms in this latter situation, using the gastric mill (chewing) CPG in the crab (Cancer borealis) stomatogastric ganglion, where stimulating the projection neuron MCN1 (modulatory commissural neuron 1) or bath applying CabPK (C. borealis pyrokinin) peptide elicits the same gastric mill motor pattern, despite configuring different gastric mill circuits. In both cases, the core rhythm generator includes the same reciprocally inhibitory neurons LG (lateral gastric) and Int1 (interneuron 1), but the pyloric (food-filtering) circuit pacemaker neuron AB (anterior burster) is additionally necessary only for CabPK rhythm generation. MCN1 drives this rhythm generator by activating in the LG neuron the modulator-activated inward current $\left(I_{\mathrm{MI}}\right)$, which waxes and wanes periodically due to phasic feedback inhibition of MCN1 transmitter release. Each buildup of $I_{\mathrm{MI}}$ enables the LG neuron to generate a self-terminating burst and thereby alternate with Int1 activity. Here we establish that CabPK drives gastric mill rhythm generation by activating in the LG neuron $I_{\mathrm{MI}}$ plus a slowly activating transient, low-threshold inward current $\left(I_{\text {Trans-LTS }}\right)$ that is voltage, time, and $\mathrm{Ca}^{2+}$ dependent. Unlike MCN1, CabPK maintains a steady $I_{\mathrm{MI}}$ activation, causing a subthreshold depolarization in LG that facilitates a periodic postinhibitory rebound burst caused by the regular buildup and decay of the availability of $I_{\text {Trans-LTS }}$. Thus, different modulatory inputs can use different rhythm-generating mechanisms to drive the same neuronal rhythm. Additionally, the same ionic current $\left(I_{\mathrm{MI}}\right)$ can play different roles under these different conditions, while different currents $\left(I_{\mathrm{MI}}, I_{\text {Trans-LTS }}\right)$ can play the same role.

\section{Introduction}

Different modulatory inputs enable individual neuronal networks to generate different output patterns by changing the intrinsic and synaptic properties of network neurons (Dickinson, 2006; Briggman and Kristan, 2008; Doi and Ramirez, 2008; Rauscent et al., 2009; Harris-Warrick, 2011; Marder, 2012). However, different modulatory inputs can also elicit the same activity pattern from that network (Saideman et al., 2007b). Determining how different modulatory pathways influence network activity is challenging, because these different pathways can converge onto the same direct inputs to a network (Di Prisco et al., 2000; Korn and Faber, 2005; Derjean et al., 2010; White and Nusbaum, 2011), comparably modulate the same network (Doi and Ramirez, 2010), distinctly alter multiple cellular and synaptic properties in the same circuit neurons (MacLean et al., 2003; Prinz et al., 2004a; Goaillard et al., 2009; Calabrese et al., 2011; Marder, 2012), and/or configure different circuits (Saideman et

\footnotetext{
Received July 29, 2013; revised Sept. 17, 2013; accepted 0ct. 7, 2013.

Author contributions: J.C.R., D.M.B., and M.P.N. designed research; J.C.R. performed research; J.C.R. and M.P.N. analyzed data; J.C.R., D.M.B., and M.P.N. wrote the paper.

This work was supported by National Institute of Neurological Disorders and Stroke Grant R37-NS 29436 (M.P.N.), National Science Foundation Grant IOS-1153417 (D.M.B.), and the Behavioral and Cognitive Neurosciences Training Grant T32-MH 17168.

Correspondence should be addressed to Dr. Michael P. Nusbaum, Department of Neuroscience, 215 Stemmler Hall, Perelman School of Medicine, University of Pennsylvania, Philadelphia, PA 19104-6074. E-mail: nusbaum@mail.med.upenn.edu.

D.M. Blitz's present address: Department of Biology, Miami University, 242 Pearson Hall, Oxford, $0 \mathrm{H} 45056$.

DOI:10.1523/JNEUROSCI.3217-13.2013

Copyright $\odot 2013$ the authors $\quad 0270-6474 / 13 / 3318047-18 \$ 15.00 / 0$
}

al., 2007b). The cellular mechanisms underlying the last of these processes are not determined in any system.

We are determining the cellular mechanisms that enable two differently configured, network-driven central pattern generator (CPG) circuits to generate the same biphasic motor pattern, using the isolated crab stomatogastric ganglion (STG; Marder and Bucher, 2007; Stein, 2009). These two gastric mill (chewing) circuits are configured by the projection neuron MCN1 (modulatory commissural neuron 1) and bath-applied CabPK (Cancer borealis pyrokinin) peptide (Saideman et al., 2007a,b).

The core rhythm generator for both gastric mill circuits includes the reciprocally inhibitory neurons LG (lateral gastric) and Int1 (interneuron 1). Rhythmic MCN1 transmitter release is also necessary for the MCN1-gastric mill rhythm, while the pyloric pacemaker neuron $\mathrm{AB}$ (anterior burster) is necessary for the CabPK rhythm. The cellular and synaptic mechanisms underlying MCN1-gastric mill rhythm generation are established (Coleman et al., 1995; Bartos et al., 1999; DeLong et al., 2009a,b). A key MCN1 rhythm-generating mechanism is its activation of the modulator-activated, voltage-dependent inward current $\left(I_{\mathrm{MI}}\right)$ in the LG neuron, which waxes and wanes periodically due to rhythmic feedback inhibition of MCN1 transmitter release by LG. These events enable the LG neuron to periodically fire a selfterminating burst and alternate with Int 1 activity.

Here we identify two CabPK-activated currents in the LG neuron that are necessary and sufficient for gastric mill rhythm generation. These currents include $I_{\mathrm{MI}}$ and a transient, lowthreshold, slowly activating inward current $\left(I_{\text {Trans-LTS }}\right) . I_{\text {Trans-LTS }}$ 
exhibits voltage- and time-dependent properties. CabPK-gastric mill rhythm generation results from $I_{\mathrm{MI}}$ providing a constant depolarizing drive that enables periodic postinhibitory rebound (PIR) bursting, triggered by $I_{\text {Trans-LTS. The }}$ rhythmic nature of the PIR burst generation results from the time- and voltagedependent properties of $I_{\text {Trans-LTS }}$. Computational modeling and dynamicclamp (DClamp) manipulations of these two currents support their necessity and sufficiency for CabPK-gastric mill rhythm generation, and reveal that the pyloric rhythm ( $\mathrm{AB})$-timed influence on the LG neuron is necessary for triggering each PIR burst. Thus, distinct rhythmgenerating mechanisms enable distinct circuits to generate the same rhythmic activity. Additionally, the same ionic current $\left(I_{\mathrm{MI}}\right)$ plays a different role under these two conditions, whereas different currents $\left(I_{\mathrm{MI}}, I_{\text {Trans-LTS }}\right)$ play a comparable role.

\section{Materials and Methods}

Animals. Male Jonah crabs (C. borealis) were purchased from commercial suppliers (Fresh Lobster, Marine Biological Laboratory) and maintained in aerated, filtered artificial seawater at $10-12^{\circ} \mathrm{C}$. Animals were cold anesthetized by packing them in ice for at least $30 \mathrm{~min}$ before dissection, after which the foregut was removed, in physiological saline at $\sim 4^{\circ} \mathrm{C}$, and the stomatogastric nervous system (STNS) isolated.

Solutions. C. borealis physiological saline contained the following (in mM): $440 \mathrm{NaCl}, 26$ $\mathrm{MgCl}_{2}, 13 \mathrm{CaCl}_{2}, 11 \mathrm{KCl}, 10$ Trisma base, 5 maleic acid, and 5 glucose, $\mathrm{pH}$ 7.4-7.6. All preparations were superfused continuously with $C$. borealis saline $\left(8-12^{\circ} \mathrm{C}\right)$. CabPK-I or CabPK-II (Saideman et al., 2007a; Biotechnology Center, University of Wisconsin, Madison, WI) was diluted from a stock solution $\left(10^{-3} \mathrm{M}\right)$ into physiological saline or voltage-clamp saline immediately before use. Bottles containing C. borealis saline and CabPK saline were connected to the same switching manifold for rapid solution changes. Oxotremorine (OXO; $10^{-5} \mathrm{M}$; Sigma), a muscarinic agonist, was applied in the same manner.

For voltage-clamp experiments, tetrodotoxin (TTX; $10^{-7}$ M; Sigma), picrotoxin (PTX; $10^{-5} \mathrm{M}$; Sigma), and tetraethylammonium chloride (TEACl; $10^{-2}$ M; Sigma) were added to $C$. borealis saline (i.e., voltageclamp saline). These substances were used to suppress voltage-dependent $\mathrm{Na}^{+}$currents (TTX), glutamatergic inhibitory synaptic transmission (PTX), and a subset of $\mathrm{K}^{+}$currents (TEACl; Marder and Eisen, 1984; Golowasch and Marder, 1992). In some experiments, the microelectrode was filled with a solution of $\mathrm{CsCl}(1 \mathrm{M}$; Sigma) and TEACl (1 M) to additionally suppress a subset of $\mathrm{K}^{+}$currents. To test the sensitivity of CabPK-influenced currents to extracellular $\mathrm{Na}^{+}$, in some experiments $\mathrm{Na}^{+}$in the saline was replaced by $\mathrm{NMDG}^{+}$( $n$-methyl, D-glucamine; Fluka; Golowasch and Marder, 1992). The $\mathrm{NMDG}^{+}$was added to the solution first and then neutralized with $\mathrm{HCl}$ before adding all other components. Additionally, in some experiments we used flufenamic acid (FFA; $10^{-5} \mathrm{M}$; Sigma), an inhibitor of $\mathrm{Ca}^{2+}$-activated, nonspecific cation current $\left(I_{\text {CAN }}\right)$, dissolved in dimethylsulfoxide (DMSO) and added directly to $C$. borealis saline. The final DMSO concentration never exceeded $1 \%$.

Electrophysiology. Electrophysiology experiments were performed using standard techniques for this system (Beenhakker and Nusbaum, 2004). In brief, the isolated STNS (Fig. 1A) was pinned into a silicone elastomer (Sylgard 184, K.R. Anderson)-lined Petri dish. Extracellular nerve recordings were obtained using pairs of stainless steel wire electrodes (reference and recording) whose ends were pressed into the Sylgard-coated dish. A differential AC amplifier (Model 1700, AM Systems) amplified the voltage difference between the reference wire, in the main bath compartment, and the recording wire, isolated with a section of an individual nerve from the main bath compartment by petroleum jelly (Vaseline, Lab Safety Supply). This signal was then further amplified and filtered (Model 410 Amplifier; Brownlee Precision). For extracellular nerve stimulation, the pair of wires used to record nerve activity was placed into a stimulus isolation unit (Model SIU5; Astromed/Grass Instruments) connected to a stimulator (Model S88; Astro-Med/Grass Instruments). 
For current-clamp experiments, intrasomatic recordings of STG neurons were made with sharp glass microelectrodes (15-30 M $\Omega$ ) filled with either $\mathrm{K}_{2} \mathrm{SO}_{4}(0.6 \mathrm{M})$ plus $\mathrm{KCl}(10 \mathrm{~mm})$ or $\mathrm{KCl}(1 \mathrm{~m})$. For voltage-clamp experiments, neurons were impaled with separate recording and current injection electrodes. The recording electrode, in most experiments, contained $\mathrm{CsCl}(1 \mathrm{M})$ and TEACl $(1 \mathrm{M})$ to suppress additional $\mathrm{K}^{+}$currents. The current injection electrode was filled with $\mathrm{KCl}(2.5 \mathrm{M})$. All intracellular recordings were amplified using Axoclamp 900A amplifiers (Molecular Devices) in bridge mode or discontinuous current-clamp mode (2-5 kHz sampling rate) and digitized at $5 \mathrm{kHz}$ using a Micro 1401 data acquisition interface and Spike2 software (Cambridge Electronic Design). To facilitate intracellular recording, the desheathed STG was viewed with light transmitted through a dark-field condenser (Nikon). In all experiments, the STG was isolated from the commissural ganglia (CoGs) by bisecting the inferior and superior oesophageal nerves (Fig. $1 A)$. Individual STNS neurons were identified by their axonal pathways, activity patterns, and interactions with other neurons (Weimann et al., 1991; Blitz et al., 1999; Beenhakker and Nusbaum, 2004).

During the gastric mill rhythm, the LG neuron burst defines the protractor phase while its interburst duration, which is equivalent to the duration of Int1 activity, defines the retractor phase (Coleman et al., 1995; Bartos et al., 1999; Diehl et al., 2013). In experiments where Int1 activity was suppressed by hyperpolarizing current injection to trigger PIR in the LG neuron, the current was usually injected into the ventricular dilator (VD) neuron instead of directly into Int 1 . These two neurons are electrically coupled, VD has a larger soma and therefore is easier to impale and manipulate, and VD has no direct synapse onto LG (Fig. 1B). Suppressing Int 1 activity via this approach was routinely confirmed by the absence of unitary IPSPs in LG, insofar as Int1 is the only source of unitary IPSPs in LG in the isolated STG. The hyperpolarizing current duration used to elicit PIR was standardized at $5 \mathrm{~s}$, which approximates the retraction phase (Intl active) duration of the gastric mill rhythm (Saideman et al., 2007b). The PIR burst was defined as having a minimum of three spikes with interspike intervals $\leq 2 \mathrm{~s}$. In the TTX experiments, the PIR response was measured as the amount of depolarization following the hyperpolarizing step relative to the baseline voltage before the step.

Two-electrode voltage-clamp. We used two-electrode voltage-clamp (TEVC) to record currents in the LG neuron. In these experiments, LG was impaled with designated recording and current-injecting electrodes. Recordings were used only if they exhibited a minimum input resistance $\left(R_{\text {input }}\right)$ of $5 \mathrm{M} \Omega$. The range of $R_{\text {input }}$ was 5-15 M . Protocols were developed and injected using pClamp software (Molecular Devices).

Modulator-activated currents were identified using two basic voltageclamp protocols, including ramps and steps. $I_{\mathrm{MI}}$ was isolated by injecting voltage ramps into LG ( -90 to $0 \mathrm{mV}$ at $75 \mathrm{mV} / \mathrm{s}$ ) in the presence of CabPK peptide $\left(10^{-6} \mathrm{M}\right)$ and saline, after which the currents recorded in control saline were subtracted from those recorded with peptide present (Swensen and Marder, 2000; DeLong et al., 2009a). In some experiments, voltage ramps were used instead to identify $I_{\mathrm{MI}}$ activated by OXO $\left(10^{-5}\right.$ M) application. $I_{\mathrm{MI}}$ was originally described by Golowasch and Marder (1992) as a proctolin-activated current and thus designated $I_{\text {proct }}$. However, many modulators are now known to activate this current in the STG (Swensen and Marder, 2000, 2001), so it is now designated as $I_{\mathrm{MI}}$ (DeLong et al., 2009a; Grashow et al., 2009).

Transient currents cannot be reliably identified with ramp protocols so, to determine whether any transient currents were influenced by CabPK, we also implemented a standard prestep voltage-clamp step protocol. To characterize CabPK-influenced transient currents, we obtained estimations of their activation $(m)$ and inactivation $(h)$ parameters by independently varying the holding voltage, prestep voltage, prestep duration, and step voltage, focusing primarily on the physiological range of LG membrane potentials $(-65$ to $-30 \mathrm{mV}$ ). To measure the voltage dependence of activation, LG was hyperpolarized to a prestep voltage of $-80 \mathrm{mV}$ for $10 \mathrm{~s}$ followed by a step depolarization to a voltage between -65 and $0 \mathrm{mV}$, in $5 \mathrm{mV}$ increments. To determine the voltage dependence of deinactivation of the CabPK-influenced transient currents, the LG neuron was held at $-45 \mathrm{mV}$ and given a hyperpolarizing prestep to $-50,-60,-70$, or $-80 \mathrm{mV}$ for $10 \mathrm{~s}$, and then stepped back to $-45 \mathrm{mV}$ for $6 \mathrm{~s}$. To measure the time dependence of deinactivation, LG was given a hyperpolarizing prestep to $-80 \mathrm{mV}$ for a range of durations $(1-13 \mathrm{~s})$ and then depolarized to $-40 \mathrm{mV}$ for $6 \mathrm{~s}$. Currents measured in normal saline were subtracted from those measured during CabPK bath application $\left(10^{-6} \mathrm{M}\right)$. In all figures, unless otherwise indicated, the subtracted currents (CabPK saline - normal saline) are displayed. We assessed the sensitivity of the $I_{\text {Trans-LTS }}$ to $\mathrm{Ca}^{2+}$ influx by replacing most of the $\mathrm{Ca}^{2+}$ in the saline with equimolar $\mathrm{Mn}^{2+}\left(0.1 \times \mathrm{Ca}^{2+}\right.$ saline). We also determined the sensitivity of $I_{\text {Trans-LTS }}$ to $\mathrm{Na}^{+}$influx by replacing $\mathrm{Na}^{+}$with an equimolar concentration of $\mathrm{NMDG}^{+}$

Dynamic clamp. We used the dynamic-clamp to inject artificial versions of ionic $\left[I_{\mathrm{MI}}\right.$, transient, low-threshold fast inward current $\left.\left(I_{\text {Trans-LTF }}\right), I_{\text {Trans-LTS }}\right]$ and synaptic (Int1-mediated inhibition) currents into the LG neuron (Sharp et al., 1993; Bartos et al., 1999; Prinz et al., 2004b; Beenhakker et al., 2005; DeLong et al., 2009a,b; DeLong and Nusbaum, 2010; Blitz and Nusbaum, 2012). The dynamic-clamp software used the intracellularly recorded LG neuron membrane potential to calculate and continually update an artificial, dynamicclamp current $\left(I_{\mathrm{dyn}}\right)$, using a predetermined reversal potential and a conductance $\left[g_{\text {dyn }}(t)\right]$ that was numerically computed. The injected current was based on real-time computations, updated in each time step $(0.2 \mathrm{~ms})$ according to the new values of recorded membrane potential, and injected back into the LG neuron. The currents were computed according to the following equations:

$$
\begin{gathered}
I_{\mathrm{dyn}}=G_{\mathrm{max}} m^{p} h^{q} n^{r}\left(V_{1}-E_{\mathrm{syn}}\right) \\
t_{x}\left(V_{2}\right) \frac{\mathrm{dX}}{\mathrm{dt}}=X_{\infty}\left(V_{2}\right)-X ; X=m, h \\
X_{\infty}(V)=\frac{1}{1+\exp \left(\frac{V-V_{x}}{k_{X}}\right)} \\
t_{X}(V)=t_{X, \mathrm{Lo}}+\frac{t_{X, \mathrm{Hi}}-t_{X, \mathrm{Lo}}}{1+\exp \left(\frac{V-V_{x}}{k_{X}}\right)}
\end{gathered}
$$

where $V_{1}$ and $V_{2}$ both represent the membrane potential, and $X$ represents either $m$ or $h$ for calculations involving activation or inactivation, respectively. The values are provided in Table 1.

We modeled our dynamic-clamp $I_{\mathrm{MI}}$ using previously determined parameters (Table 1; Golowasch and Marder, 1992; Swensen and Marder, 2000, 2001; DeLong et al., 2009a). Specifically, we set the half-maximum voltage of the activation curve at $-42 \mathrm{mV}$, with the peak current occurring at $-32 \mathrm{mV}$ and the reversal potential $\left(E_{\text {syn }}\right)$ at $0 \mathrm{mV}$. These values reflect the ones obtained from intraneurite $\mathrm{LG}$ neuron recordings within the STG neuropil (DeLong et al., 2009a). Hence, they occur at more hyperpolarized potentials than those obtained in the current study from intrasomatic recordings, which are electrotonically more distant from the site of these events within the neuropil. $I_{\mathrm{MI}}$ shows a voltage dependence to its activation (Golowasch and Marder, 1992; Swensen and Marder, 2000, 2001). Therefore, the integer power of the activation variable $m(P)$ was set to a value of 1 . The slope of the activation curve $\left(K_{\mathrm{m}}\right)$ was $-5.0 \mathrm{mV}$, and the time constant of activation was $5.0 \mathrm{~s}^{-1} . I_{\mathrm{MI}}$ does not inactivate, so the integer power of the inactivation variable $h$ (abbreviated $q$ above) was set to 0 . The conductance value at maximum activation $\left(G_{\max }\right)$ varied between 50 and $200 \mathrm{nS}$. In all of our dynamic-clamp experiments, the maximum current injected into the LG neuron never exceeded $3 \mathrm{nA}$ (see Results). Synaptic conductances were modeled in a manner similar to intrinsic conductances, except that activation depended on the presynaptic neuron voltage and was more depolarized than the presynaptic action potential threshold. These synapses have been well documented and incorporated into previous models of gastric mill rhythm generation (Nadim et al., 1998; Bartos et al., 1999; Kintos et al., 2008; DeLong and Nusbaum, 2010; Blitz and Nusbaum, 2012), and the $G_{\max }$ could be readily scaled to match the observed physiological synapses.

Our dynamic-clamp model for both of the low-threshold transient currents was based on the aforementioned voltage-clamp step protocol 
Table 1. Dynamic-clamp conductances

\begin{tabular}{|c|c|c|c|c|c|c|c|c|c|}
\hline Neuron & Conductances & Parameters & $V_{1 / 2}(\mathrm{mV})$ & $k(\mathrm{mV})$ & $t_{\mathrm{to}}(\mathrm{ms})$ & $t_{\mathrm{hi}}(\mathrm{ms})$ & $\operatorname{Exp}(p, q, r)$ & $E_{\mathrm{rev}}$ & $G_{\max }$ \\
\hline \multirow[t]{3}{*}{ LG (biological) } & \multirow[t]{2}{*}{$I_{\text {Trans-LTS }}$} & $m$ & -50 & -3 & 500 & 500 & 1 & \multirow[t]{2}{*}{10} & \multirow[t]{2}{*}{0.6} \\
\hline & & $h$ & -58 & 0.8 & 3500 & 1500 & 1 & & \\
\hline & $I_{\mathrm{MI}}$ & $m$ & -50 & -5 & 5 & 5 & 1 & 10 & 0.1 \\
\hline \multirow[t]{5}{*}{ Int1 (model) } & \multirow[t]{2}{*}{$\mathrm{Na}_{\mathrm{v}}$} & $m$ & -42 & -5 & 1.5 & 0.45 & 3 & \multirow[t]{2}{*}{45} & \multirow[t]{2}{*}{7.5} \\
\hline & & $h$ & -52 & 9.2 & 10 & 2.4 & 1 & & \\
\hline & \multirow[t]{2}{*}{$K_{\mathrm{v}}$} & $m$ & -26 & -9 & 27 & 3 & 4 & \multirow[t]{2}{*}{-70} & \multirow[t]{2}{*}{30} \\
\hline & & $h$ & -16 & 1.5 & 20 & 200 & 2 & & \\
\hline & Leak & & & & & & & -60 & 0.012 \\
\hline $\operatorname{lnt1}>$ LG & Synapse & $m$ & -40 & -1 & 200 & 200 & 1 & -80 & 2 \\
\hline $\mathrm{LG}>\operatorname{lnt} 1$ & Synapse & $m$ & -30 & -0.1 & 200 & 200 & 1 & -80 & 15 \\
\hline
\end{tabular}

$V_{1 / 2}$, half-activation voltage; $E_{\text {rev }}$, reversal potential.

experiments. The results from activation protocols were manually fit to Hodgkin-Huxley equations using HHfit (version 3.2) software developed by the Nadim laboratory [New Jersey Institute of Technology (NJIT) and Rutgers University, Newark, NJ; available at http://stg. rutgers.edu/software/]. Occasionally, the resting $V_{\mathrm{m}}$ and action potential threshold coordinately varied between preparations, possibly due to impalement quality. Therefore, the dynamic-clamp parameters were linked to the resting $V_{\mathrm{m}}$. Table 1 contains a full parameter set for a neuron resting at $-60 \mathrm{mV}$.

We used two versions of the dynamic-clamp on a personal computer (PC) running Windows XP/7 and a NI PCI-6070-E data acquisition board (National Instruments). The first version was developed in the Nadim laboratory (NJIT and Rutgers University, Newark, NJ; available at http://stg.rutgers.edu/software/). The second version was developed by E. Brady Trexler (Fishberg Department of Neuroscience, Mt. Sinai School of Medicine; freely available through Gotham Scientific: http://gothamsci.com/NetClamp/). Dynamic-clamp current injections were performed while recording in single-electrode, DCC mode (sampling rates, $2-5 \mathrm{kHz}$ ) or with separate voltage recording and currentinjecting electrodes.

Data analysis. Data were collected onto a computer, with later playback onto a chart recorder (Everest; Astro-Med). Acquisition onto the computer (sampling rate, $5 \mathrm{kHz}$ ) used the Spike2 data acquisition and analysis system (Cambridge Electronic Design). Some analyses, including CabPK-gastric mill rhythm parameters, were conducted on the digitized data using a custom-written Spike2 program (The Crab Analyzer: freely available at http://www.neurobiologie.de/spike2).

Voltage-clamp data analysis was performed using PClamp (version 9; Molecular Devices), Spike2 (CED), and Igor Pro (Wavemetrics) software. For ramps, total neuron currents were determined by averaging 10 ramps in each condition and subtracting the control from the experimental condition. For prestep protocols, the protocols were run once in each condition and the control currents were subtracted before analysis.

For gastric mill rhythm analyses, unless otherwise stated, each data point in a dataset was derived by determining the mean for the analyzed parameter from 10 consecutive gastric mill cycles. One gastric mill cycle was defined as extending from the onset of consecutive LG neuron action potential bursts (Beenhakker and Nusbaum, 2004; Wood et al., 2004). Thus, the gastric mill cycle period was measured as the duration (in seconds) between the onset of two successive LG neuron bursts. The protractor phase was measured as the LG neuron burst duration, while the retractor phase was measured as the LG neuron interburst duration. The gastric mill rhythm-timed LG neuron burst duration was defined as the duration (in seconds) between the onset of the first and last action potential within an impulse burst, during which no interspike interval was $>1.5 \mathrm{~s}$ (approximately one pyloric cycle period during the CabPK-gastric mill rhythm and briefer than the duration of each gastric mill phase; Saideman et al., 2007b). The intraburst firing rate of the LG neuron was defined as the number of action potentials -1 , divided by the burst duration.

Data were plotted with Igor Pro (version 6.10A). Figures were produced using CorelDraw (version 13.0 for Windows). Statistical analyses were performed with Microsoft Excel and SigmaStat 3.0 (SPSS). Comparisons were made to determine statistical significance using the paired Student's $t$ test or ANOVA with repeated-measures followed by the Stu-
dent-Newman-Keuls (SNK) post hoc test. In all experiments, the effect of each manipulation was reversible, and there was no significant difference between the premanipulation and postmanipulation groups. Data are expressed as the mean $\pm \mathrm{SE}$.

Gastric mill model. We constructed a computational model of the CabPK-gastric mill rhythm generator modified from an existing conductance-based model of the MCN1-gastric mill rhythm generator (Nadim et al., 1998; Beenhakker et al., 2005; DeLong et al., 2009a,b). The previously published version modeled the LG, Int1, and MCN1 neurons as having multiple compartments separated by an axial resistance, with each compartment possessing intrinsic and/or synaptic conductances. The parameters of the CabPK-gastric mill rhythm generator model were based on both previously published voltage-clamp analyses in STG neurons (including LG) and on the LG neuron voltage-clamp results obtained in this study (Golowasch and Marder, 1992; Swensen and Marder, 2000, 2001; DeLong et al., 2009a). To mimic the effects of CabPK bath application to the biological system, we added $I_{\mathrm{MI}}$ to the LG neuron dendrite compartment as an intrinsic (nonsynaptically activated) current (Table 2). This approach was based on the fact that CabPK excites LG by activating $I_{\mathrm{MI}}$ (this study) and that CabPK was constantly present during its application. To more realistically mimic the biological system, in this version of the model we modified the CabPK-activated $G_{\mathrm{MI}}$ in the LG neuron dendrite compartment to include a voltage dependence ( $\mathrm{Ta}-$ ble 2). Based on data collected in this study, we also added a CabPKactivated $I_{\text {Trans-LTS }}$ to the LG neuron (Table 2). The time and voltage dependence of $I_{\text {Trans-LTS }}$ were empirically determined in the present study with voltage-clamp analyses.

Simulations were performed on a PC with the freely available Ubuntu Linux operating system (www.ubuntu.com). We used the Network simulation software developed in the Nadim laboratory (http://stg.rutgers. edu/software/network.htm). This included using a fourth-order RungeKutta numerical integration method with time steps of 0.05 and $0.01 \mathrm{~ms}$. Results were visualized by plotting outputted data points using the freely available Gnuplot software package (www.gnuplot.info). In most figures showing the model output, we present conductance $(g)$ instead of the associated current $(I)$ to more clearly display the trajectory during the gastric mill retractor and protractor phases. The main difference between $g$ and $I$ is that the former lacks the fast transient changes that occur in the latter during each LG neuron action potential (DeLong et al., 2009a). In particular, the relatively slow kinetics of the CabPK-activated conductances make them insensitive to these fast transient changes in voltage.

The presentation of currents in the model and dynamic-clamp figures represent different conventions. Specifically, the model output uses the standard voltage-clamp convention, whereas the dynamic-clamp output uses the standard current-clamp convention. For example, depolarizing current has a downward trajectory in the model output figures but has an upward trajectory in the dynamic-clamp output figures.

\section{Results}

In the isolated crab STG, tonic MCN1 stimulation and bathapplied CabPK $\left(\geq 10^{-7} \mathrm{M}\right)$ elicit comparable gastric mill motor patterns, despite configuring different gastric mill circuits (Fig. 1B; Saideman et al., 2007b). MCN1 does not contain CabPK, and 
Table 2. CabPK- gastric mill network model parameters

\begin{tabular}{|c|c|c|c|c|c|c|c|c|c|}
\hline Neuron & Conductances & Parameters & $V_{1 / 2}(\mathrm{mV})$ & $k(\mathrm{mV})$ & $t_{\mathrm{to}}(\mathrm{ms})$ & $t_{\mathrm{hi}}(\mathrm{ms})$ & $\operatorname{Exp}(p, q, r)$ & $E_{\mathrm{rev}}$ & $G_{\max }$ \\
\hline \multirow[t]{3}{*}{ LG } & \multirow[t]{2}{*}{$I_{\text {Trans-LTS }}$} & $m$ & -55 & -3 & 500 & 500 & 1 & & \\
\hline & & $h$ & -63 & 0.8 & 3500 & 1500 & 1 & & \\
\hline & $I_{\mathrm{MI}}$ & $m$ & -60 & -5 & 5 & 5 & 1 & & \\
\hline \multirow[t]{5}{*}{ Int1 } & \multirow[t]{2}{*}{$\mathrm{Na}_{\mathrm{v}}$} & $m$ & -42 & -5 & 1.5 & 0.45 & 3 & 45 & 7.5 \\
\hline & & $h$ & -52 & 9.2 & 10 & 2.4 & 1 & & \\
\hline & \multirow[t]{2}{*}{$K_{\mathrm{V}}$} & $m$ & -26 & -9 & 27 & 3 & 4 & -70 & 30 \\
\hline & & $h$ & -16 & 1.5 & 20 & 200 & 2 & & \\
\hline & Leak & & & & & & & -60 & 0.012 \\
\hline $\operatorname{lnt} 1>\mathrm{LG}$ & Synapse & $m$ & -40 & -1 & 200 & 200 & 1 & -80 & 2 \\
\hline $\mathrm{LG}>\operatorname{lnt} 1$ & Synapse & $m$ & -30 & -0.1 & 200 & 200 & 1 & -80 & 15 \\
\hline
\end{tabular}

$V_{1 / 2}$, half-activation voltage; $E_{\text {rev }}$, reversal potential.

A Normal Saline

$\mathrm{LG}_{-58.1 \mathrm{mV}}$

D Normal Saline

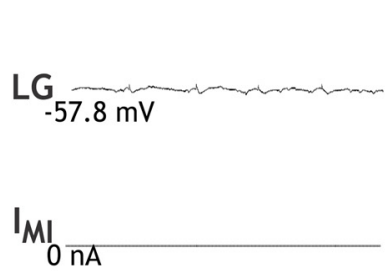

CabPK $\left(10^{-6} \mathrm{M}\right)$

$-49.8 \mathrm{mV}$
$0.5 \frac{\mathrm{nA}}{2 \mathrm{~s}}$

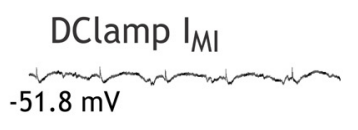

$-51.8 \mathrm{mV}$

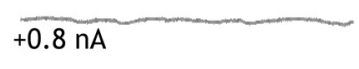

$0.5 \frac{\mathrm{mA}}{2 \mathrm{~s}}$
B CabPK $\left(10^{-6} \mathrm{M}\right)$ onset
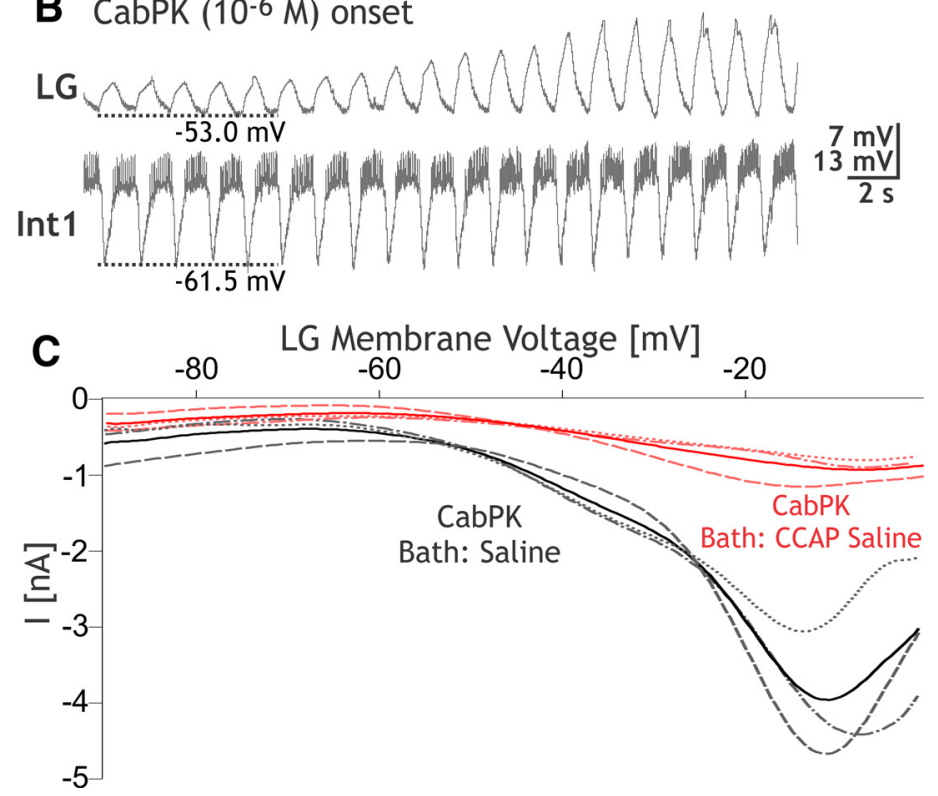

Figure 2. Bath-applied CabPK peptide elicits a sustained, subthreshold depolarization in the isolated LG neuron by activating the voltage-dependent $I_{\text {MI }} \cdot A$, With $I n t 1$ neuron activity suppressed by hyperpolarizing current injection, bath-applied CabPK elicited a sustained, subthreshold depolarization in the LG neuron. B. With Int1 neuron exhibiting its normal pyloric-timed burst pattern, thereby providing rhythmic inhibition to LG, bath-applied CabPK initially caused a gradual increase in the amplitude of the subthreshold, pyloric-timed oscillations in LG. Note that the oscillation peaks became more depolarized, while the membrane potential of the trough was not changed. CabPK superfusion was begun immediately before the start of this trace. Subsequently, the gastric mill rhythm commenced. C, I-V plots of CabPK-influenced current in LG, obtained using TEVC and a voltage ramp protocol, during focal pressure application (5 psi, $1 \mathrm{~s})$ of CabPK (10 $\left.{ }^{-4} \mathrm{M}\right)$ under control conditions (black) and during (CAP $\left(10^{-6} \mathrm{M}\right)$ bath application (red). Each curve represents the difference current (CabPK - control or CCAP condition) as indicated. Solid curves represent the mean values for each condition; broken lines represent three individual experiments. $\boldsymbol{D}$, Injection of artificial $I_{\mathrm{MI}}\left(g_{\mathrm{MI}}=100 \mathrm{nS}\right)$ into LG, via the dynamic-clamp, in a preparation where Int 1 activity was weak $(<5 \mathrm{~Hz})$ caused a sustained depolarization comparable to that resulting from CabPK application.

the CabPK-gastric mill rhythm can occur without MCN1 activity. CabPK is present in two or three pairs of CoG projection neurons that innervate the STG, although these neurons are not physiologically identified (Saideman et al., 2007a). However, bath-applied peptide can mimic the actions resulting from its neuronal release. For example, in the crab STG, bath application of the peptide proctolin $\left(10^{-6} \mathrm{M}\right)$ and direct stimulation of the modulatory proctolin neuron (MPN) elicit comparable responses from the pyloric CPG, despite the fact that MPN contains a small-molecule cotransmitter (Nusbaum and Marder, 1989a,b; Blitz et al., 1999).

There are also at least several additional gastric mill motor patterns in C. borealis, each distinct from the pattern elicited by MCN1 and CabPK, and driven by a different input pathway (Beenhakker and Nusbaum, 2004; Blitz et al., 2004; Christie et al., 2004; White and Nusbaum, 2011). These different rhythms all share the same basic structure, which includes a biphasic motor pattern exhibiting rhythmic alternating bursting of protraction- and retraction-related neurons across an overlapping range of cycle periods ( $\sim-20 \mathrm{~s}$; Fig. $1 C)$. They differ in the relative timing, intensity, duration, and pattern of activity in the component neurons. There are seven gastric mill motor neurons, including four protractor motor neurons and three retractor motor neurons, plus a single retraction-timed interneuron (Int1; Fig. 1B). As discussed below, the pyloric CPG pacemaker neuron $\mathrm{AB}$ also influences these gastric mill rhythms (Fig. 1B). Like the gastric mill rhythm, the pyloric (filtering of chewed food) rhythm is generated in the STG (Marder and Bucher, 2007).

A core component of the rhythm generator for the MCN1and CabPK-gastric mill rhythms is the half-center formed by the reciprocally inhibitory protraction neuron LG and retraction neuron Int1 (Saideman et al., 2007b; Fig. 1B). The biphasic rhythm generated by these two neurons is then imposed on the other gastric mill neurons by synaptic actions from the rhythm generator plus the influences of MCN1 or CabPK. Under baseline conditions, the LG neuron is silent (Fig. 1C), and Int1 is sponta- 
neously active, exhibiting a pyloric rhythm-timed activity pattern due to inhibitory input that it receives from the $\mathrm{AB}$ neuron (Fig. 1B; Bartos et al., 1999; Saideman et al., 2007b). The pivotal event for enabling gastric mill rhythm generation is the acquisition by the LG neuron of the ability to fire rhythmic bursts.

The cellular and synaptic mechanisms underlying MCN1 activation of the gastric mill rhythm generator are known (Coleman et al., 1995; Bartos et al., 1999; DeLong et al., 2009a). In brief, during the MCN1-gastric mill rhythm, there is a rhythmic release of the MCN1 cotransmitters, which include the peptides proctolin and CabTRP Ia (C. borealis tachykininrelated peptide Ia) plus GABA (Blitz et al., 1999). MCN1 uses only CabTRP Ia to influence LG (slow excitation) and only GABA to influence Intl (fast excitation; Wood et al., 2000; Stein et al., 2007). MCN1 cotransmitter release is rhythmic, even when MCN1 is tonically active, because its STG terminals $\left(\mathrm{MCN1}_{\mathrm{STG}}\right)$ receive ionotropic synaptic inhibition from LG (Fig. 1B; Coleman and Nusbaum, 1994). Thus, during retraction, continuous MCN1 release of CabTRP Ia drives a steady buildup of $I_{\mathrm{MI}}$ in LG that eventually is sufficient to enable LG to fire an action potential burst (DeLong et al., 2009a). During protraction, when $\mathrm{MCN1}_{\mathrm{STG}}$ cotransmitter release is inhibited by $\mathrm{LG}$, there is a steady decline in the amount of $I_{\mathrm{MI}}$ in LG until it can no longer sustain the LG neuron burst. This rhythmic activation of $I_{\mathrm{MI}}$ in LG appears to be sufficient to drive the gastric mill rhythm across the physiological range of MCN1 firing frequencies (DeLong et al., 2009a,b; DeLong and Nusbaum, 2010). MCN1-driven gastric mill rhythm generation is also facilitated by the pyloric rhythm (cycle period, $\sim 1 \mathrm{~s}$ ), because every LG neuron burst initiates, after sufficient $I_{\mathrm{MI}}$ has accrued, during a pylorictimed depolarization (i.e., disinhibition) that results from $\mathrm{AB}$ neuron inhibition of Int1 (Bartos et al., 1999; DeLong et al., 2009a). These disinhibitions reduce the $\mathrm{MCN1}$-gastric mill cycle period by reducing the amount of $I_{\mathrm{MI}}$-mediated depolarization needed to enable LG to fire a burst. However, this rhythm does persist, with a longer cycle period, when there is no pyloric rhythm (Bartos et al., 1999).

The cellular and synaptic mechanisms underlying the CabPKgastric mill rhythm were not known, although it was determined previously that activity in LG, Int 1 , and AB was necessary to enable this rhythm (Saideman et al., 2007b). Additionally, as during the MCN1-gastric mill rhythm, it appeared that direct CabPK excitation of the LG neuron was a pivotal event for rhythm generation. Thus, we identified CabPK-influenced ionic currents in LG.
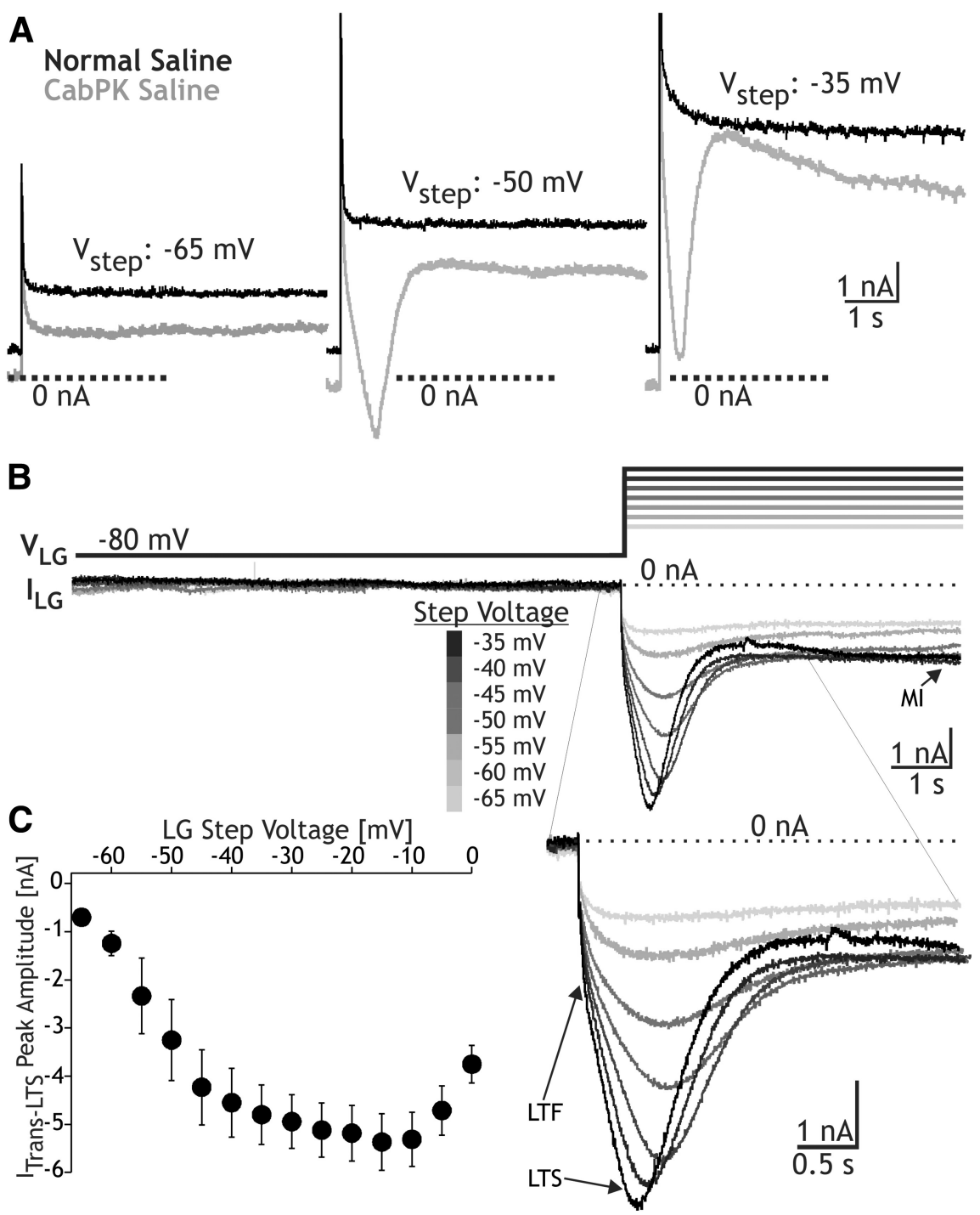

Figure 3. CabPK application influences both transient and sustained voltage-dependent inward currents in the $L G$ neuron. $A$, Example raw current traces recorded during TEVC using a voltage step protocol $\left(V_{\text {hold }}=-80 \mathrm{mV}\right)$ to the indicated step potentials, during superfusion with voltage-clamp saline under control and CabPK conditions (see Materials and Methods). Note that, during the two more depolarized steps ( -50 and $-35 \mathrm{mV}$ ) in the presence of (abPK, there was a relatively large amplitude transient inward current, while during all three steps there was a reduction in the amplitude of the sustained outward current relative to the control condition. B, Top, CabPK-influenced currents (CabPK condition - control condition) resulting from a voltage step protocol during TEVC included a low threshold, fast transient current $\left(I_{\text {Trans-LTF }}\right), I_{\text {Trans-LTS }}$, and sustained current $\left(I_{M \mathrm{M}}\right) \cdot I_{\text {Trans-LTS }}$ was predom$I_{\text {Trans-LTF }}$ did not exhibit a distinct, separate peak in this experiment (Figs. $4 A, 5 A$ ), but is evident as an initial steep inward slope before the shallower rising slope representing I $_{\text {Trns-ITs. }}$. Entire voltage step protocol (hold at $-80 \mathrm{mV}, 10 \mathrm{~s}$; step to test voltage, $6 \mathrm{~s}$ ) is shown. Bottom, Expansion of the current traces to highlight the events occurring during each voltage step. C, I-V plot of the current (mean $\pm \mathrm{SE}$ ) at the peak of $/_{\text {Trans-LTS }}$ for the step protocol used in experiments such as that in $\boldsymbol{B}(n=9)$.

CabPK activates three voltage-dependent inward currents in the LG neuron

CabPK application $\left(10^{-6} \mathrm{M}\right)$ provides a depolarizing drive to $\mathrm{LG}$ from its resting potential $(-57.5 \pm 1.5 \mathrm{mV} ; n=8)$. For example, under the most reduced conditions, with LG isolated from synaptic input, CabPK consistently elicited in LG a steady 5-10 mV depolarization. This depolarizing response occurred when LG was isolated by either hyperpolarizing Int1 (depolarizing response, $8.5 \pm 1.1 \mathrm{mV} ; n=8$; Fig. $2 A$ ) or suppressing all glutamatergic inhibitory input to LG by bath-applying PTX $\left(10^{-5} \mathrm{M}\right.$; depolarizing response, $10.7 \pm 0.54 \mathrm{mV} ; n=11$ ). The CabPKmediated depolarization moved the LG neuron membrane 
potential closer to its spike threshold, which was not changed by CabPK (saline, $-42.4 \pm 1.3 \mathrm{mV} ; n=9$; CabPK, $-44.5 \pm 1.4 \mathrm{mV}$; $n=9, p=0.15)$. When Int 1 was active, the LG neuron membrane potential exhibited subthreshold, pyloric-timed oscillations that exhibited a more depolarized peak in the presence of CabPK (saline, $-57.5 \pm 1.4 \mathrm{mV} ; n=10$; CabPK, $-48.7 \pm 1.7 \mathrm{mV} ; n=$ $10, p<0.01$; Fig. $2 B$ ). These depolarized peaks remained subthreshold before the onset of the gastric mill rhythm, as well as during the ensuing gastric mill retraction phase. In contrast, CabPK did not alter the membrane potential at the trough of these LG neuron oscillations (saline, $-59.8 \pm 1.8 \mathrm{mV} ; n=10$; CabPK, $-59.1 \pm 1.7 \mathrm{mV} ; n=10, p=0.4$; Fig. $2 B$; see below). Thus, the increased pyloric-timed oscillation amplitude was due to a more depolarized peak.

Based on the assumption that the sustained depolarizing drive in the LG neuron during CabPK application resulted from CabPK influence on a persistent current, we isolated CabPKsensitive currents using a voltage ramp protocol (see Materials and Methods) in TEVC. Difference currents between CabPK and control solutions revealed a voltage-dependent, inward net current at potentials more hyperpolarized than $0 \mathrm{mV}$ (Fig. 2C). This inward current exhibited a small, relatively constant amplitude at membrane potentials more hyperpolarized than approximately $-60 \mathrm{mV}$, whereas in the depolarizing direction from approximately $-60 \mathrm{mV}$, the $I-V$ plot for this current displayed a trajectory reminiscent of voltage-dependent inward currents. Specifically, it displayed a steadily increasing inward current that peaked at $-8.1 \pm 1.4 \mathrm{mV}$ (peak amplitude: $-4.4 \pm 0.6 \mathrm{nA}, n=$ 7 ), after which the amplitude steadily decreased (Fig. $2 C$ ). It was not possible to determine its reversal potential, likely due to an inability to completely clamp the residual, relatively large $\mathrm{K}^{+}$ currents at more depolarized potentials (DeLong et al., 2009a). This $I-V$ relationship was comparable to that of the previously identified $I_{\mathrm{MI}}$, which is activated by several different neuromodulators in crab STG neurons including the LG neuron (Golowasch and Marder, 1992; Swensen and Marder, 2000, 2001; DeLong et al., 2009a).

To further establish that the CabPK-activated, voltagedependent inward current in the LG neuron that we identified in voltage ramp protocols was $I_{\mathrm{MI}}$, we performed an occlusion experiment with a known $I_{\mathrm{MI}}$ activator in the LG neuron, crustacean cardioactive peptide (CCAP; DeLong et al., 2009a). CCAP application occludes the ability of the MCN1 peptide CabTRP Ia to activate $I_{\mathrm{MI}}$ in LG (DeLong et al., 2009a). In these experiments, CabPK $\left(10^{-4} \mathrm{M}\right)$ was first pressure applied onto the desheathed STG neuropil while recording LG in TEVC. CCAP $\left(10^{-6} \mathrm{M}\right)$ was then bath applied to activate $I_{\mathrm{MI}}$, during which time CabPK $\left(10^{-4} \mathrm{M}\right)$ was again puffed onto the STG neuropil. As shown in Figure $2 C$, the maximal CabPK-activated current amplitude was decreased substantially during CCAP bath application (CabPK pre-CCAP: $-4.2 \pm 0.6 \mathrm{nA}$; CabPK during CCAP application: $-0.9 \pm 0.1 \mathrm{nA}$; CabPK post-CCAP: $-3.5 \pm 0.9 ; p=0.01: \mathrm{CabPK}$ pre-CCAP or CabPK post-CCAP vs CabPK during CCAP: $p=$ 0.33, CabPK pre-CCAP vs CabPK post-CCAP; one-way repeated-measures ANOVA with SNK post hoc test; $n=3, F_{(2,8)}$ $=16.8$ ). This occlusion effect of CCAP thus supported the hypothesis that the aforementioned CabPK-activated inward current in the LG neuron was $I_{\mathrm{MI}}$. In contrast to the CabPK condition, in normal saline $I_{\mathrm{MI}}$ was either not expressed or was present at low levels, insofar as the LG neuron resting potential in normal saline was approximately $-60 \mathrm{mV}$ (see above), and even modest levels of modulator-activated $I_{\mathrm{MI}}$ elicit a more depolar-
A

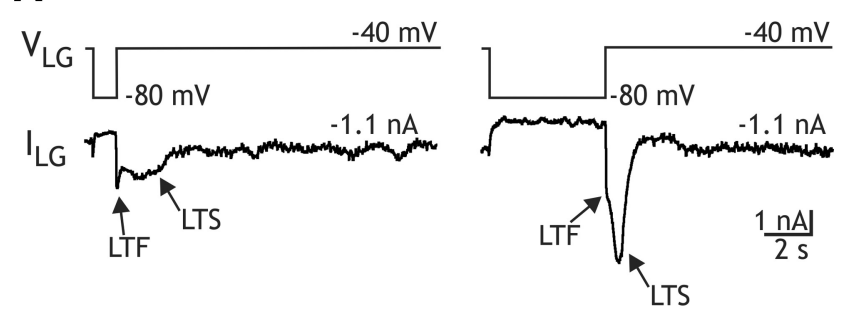

B

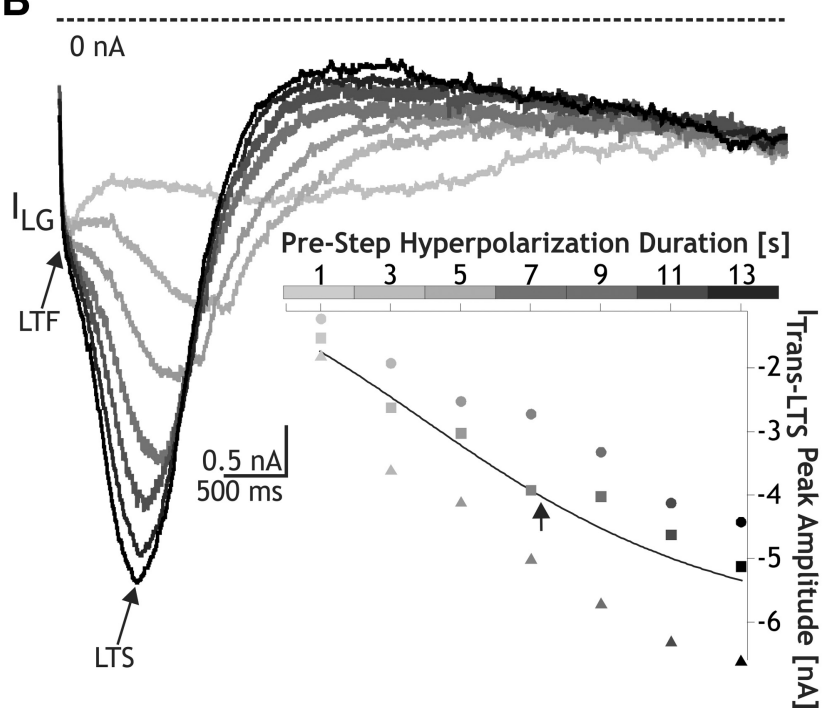

Figure 4. CabPK-activated $I_{\text {Trans-LTS }}$ in the $L G$ neuron exhibits a time-dependent deinactivation. $A$, Using TEVC, hyperpolarizing voltage steps to $-80 \mathrm{mV}$ were injected into the $L G$ neuron for relatively brief (left) and long (right) durations, and then returned to $-40 \mathrm{mV}$. A larger peak $I_{\text {Trans-LTs }}$ amplitude resulted upon the return to $-40 \mathrm{mV}$ after the longer duration hyperpolarization. In contrast, there was little change in the peak amplitude of $I_{\text {Trans-LTF }}$ for both step durations. $\boldsymbol{B}$, Superimposed series of current responses in the $L G$ neuron, aligned to the return to $-40 \mathrm{mV}$ after hyperpolarizing steps of different durations to $-80 \mathrm{mV}$ (see the gray scale, representing the different step durations, aligned with the $x$-axis of the inset scatter plot). Inset, Plot of $I_{\text {Trans-LTS }}$ peak amplitude as a function of hyperpolarizing step duration from three experiments. Each symbol represents a different experiment. The line represents the sigmoid fit to the data (Igor Pro), and the arrow represents the calculated midpoint.

ized LG neuron membrane potential (Kirby and Nusbaum, 2007; DeLong et al., 2009a).

$I_{\mathrm{MI}}$ is also sensitive to changes in extracellular $\mathrm{Ca}^{2+}$ (Golowasch and Marder, 1992). Specifically, replacing most of the extracellular $\mathrm{Ca}^{2+}\left(0.1 \times\right.$ normal $\left.\mathrm{Ca}^{2+}\right)$ in the saline with additional $\mathrm{Mg}^{2+}$, to maintain the total divalent cation concentration, linearizes the $I-V$ curve for $I_{\mathrm{MI}}$ at hyperpolarized potentials. This linearization in reduced $\mathrm{Ca}^{2+} /$ added $\mathrm{Mg}^{2+}$ saline also occurred for the CabPK-sensitive current (current measured at $-90 \mathrm{mV}$ : CabPK saline, $-0.21 \pm 0.3 \mathrm{nA}$; CabPK with reduced $\mathrm{Ca}^{2+}$ saline, $-5.67 \pm 1.3 \mathrm{nA} ; n=3, p<0.05)$, further supporting the hypothesis that CabPK activates $I_{\mathrm{MI}}$ in LG neuron.

To test the hypothesis that $I_{\mathrm{MI}}$ was responsible for the aforementioned, CabPK-mediated depolarization in LG, we used the DClamp to inject an artificial version of $I_{\mathrm{MI}}$ into LG in normal saline (Fig. 2D). Doing so using DClamp conductances comparable to those identified in voltage-clamp (50-300 nS), while Int1 was silent or only weakly active, consistently depolarized the LG neuron resting potential to the same extent as CabPK application (CabPK: $10.68 \pm 0.5 \mathrm{mV}$; DClamp $I_{\mathrm{MI}}: 10.3 \pm 2 \mathrm{mV} ; n=6, p=$ 0.4 ; Fig. $2 A, D)$. 
CabPK also activated other voltagedependent inward currents in the LG neuron. These additional currents were not evident with our voltage ramp protocol, but they were present during a TEVC voltage step protocol. Their absence during our voltage ramp manipulations was likely due to their time-dependent inactivation (see below). We identified these other currents using a prestep hyperpolarization $(-80 \mathrm{mV})$ whose duration was similar to the gastric mill retraction phase (see Materials and Methods). Using this approach, with relatively prolonged depolarizing steps (6 s) comparable to the gastric mill protraction phase during which LG is depolarized and spiking, we identified three inward currents (Figs. 3, 4, 5). These currents included (1) $I_{\text {Trans-LTF, }}$ (2) $I_{\text {Trans-LTS }}$, and (3) sustained inward current. The $I_{\text {Trans-LTF }}$ was not evident in the raw current recordings obtained during CabPK superfusion (Fig. 3A), due to overlap with the capacitative current, but was readily evident in the difference current traces (Figs. $3 B, 4,5$ ). In contrast, $I_{\text {Trans-LTS }}$ was identifiable in both the raw CabPK and difference currents (Figs. 3, 4, 5), but was not evidently expressed under control conditions (Fig. 3A). Last, in the raw CabPK recordings the sustained inward current was evident as a smaller amplitude outward current relative to the control recordings (Fig. 3A).

The CabPK-activated, sustained inward current was predominantly $I_{\mathrm{MI}}$. During the last $3 \mathrm{~s}$ of the voltage step, we consistently observed a voltage-dependent, time-independent inward current, as anticipated from our voltage ramp experiments that identified CabPK activation of $I_{\mathrm{MI}}$ (Fig. $3 B$ ). There were three features of this sustained current in the voltage step protocols, however, that were distinct from $I_{\mathrm{MI}}$ measured from the voltage ramp protocol, as follows: (1) the peak amplitude was smaller (step: $-1.6 \pm 0.4 \mathrm{nA}$, $n=9$; ramp: $-4.6 \pm 0.4 \mathrm{nA} n=9, p<0.01$ ); (2) the current exhibited less voltage dependence at depolarized potentials (data not shown); and (3) in some recordings, particularly with steps more depolarized than $-40 \mathrm{mV}$, a reduced inward current or small outward current was evident immediately following $I_{\text {Trans-LTS }}$, relative to the current amplitude at the end of the step (Figs. $3 A, B, 4 B, 5 B$ ). These features suggested that CabPK also activated a voltage- and time-dependent outward current. We did not, however, further isolate and characterize this additional component insofar as it did not appear to be necessary for the CabPK actions on gastric mill rhythm generation (see below).

The fast transient inward current exhibited a relatively rapid time to peak $(32.9 \pm 1.9 \mathrm{~ms} ; n=16)$ and small peak amplitude $(\sim 0.5 \mathrm{nA})$, which occurred at approximately $-10 \mathrm{mV}$. It exhibited an apparent voltage threshold of approximately $-45 \mathrm{mV}$ (range, -50 to $-30 \mathrm{mV} ; n=16$ ). Only an approximate peak current amplitude is provided for $I_{\text {Trans-LTF }}$ because we could not
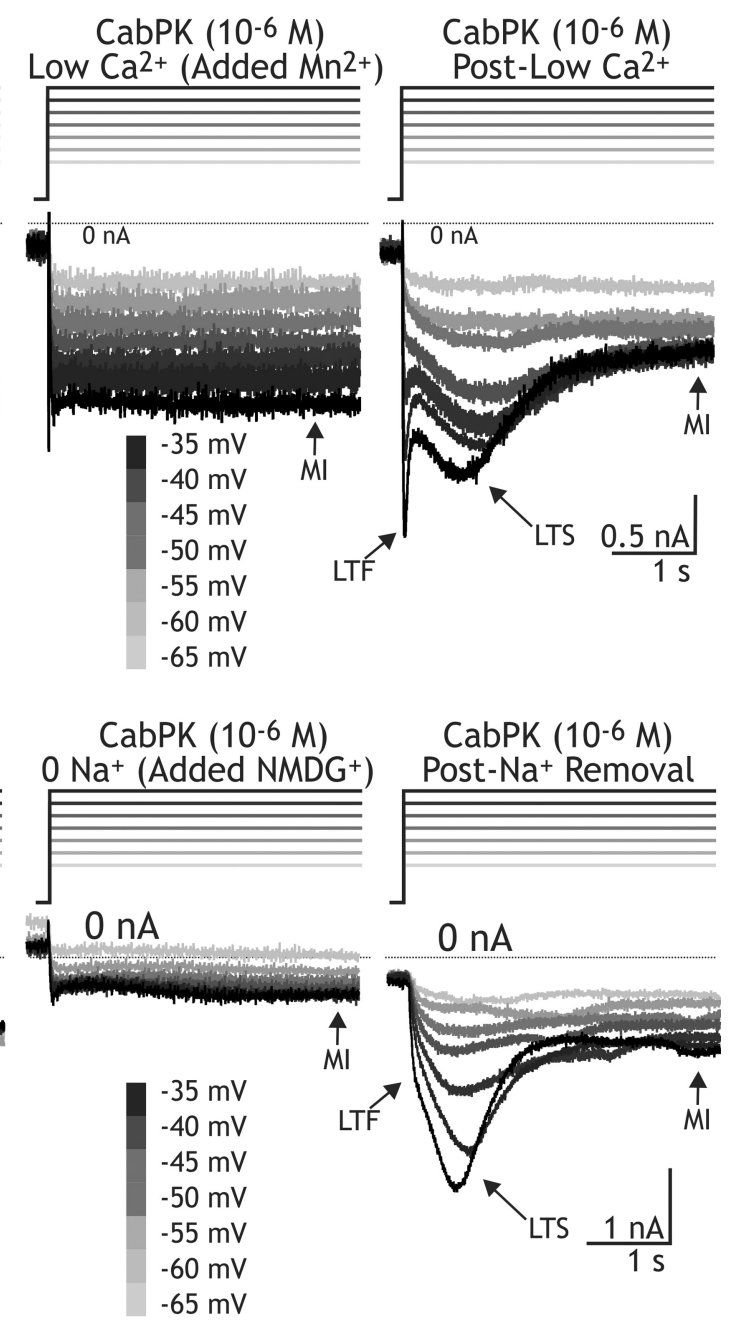

Figure 5. CabPK-activated $I_{\text {Trans-LTS }}$ is sensitive to changes in extracellular $\mathrm{Ca}^{2+}$ and extracellular $\mathrm{Na}^{+}$. $\boldsymbol{A}$, Superimposed sweeps of current recordings from the $\mathrm{LG}$ neuron, recorded in TEVC with TEACl/CsCl-filled electrodes, during depolarizing steps to different membrane potentials ( -65 to $-35 \mathrm{mV}$ ) from a holding voltage of $-80 \mathrm{mV}$, during CabPK application in control,

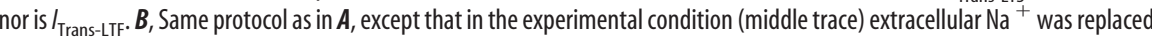
with an equimolar concentration of NMDG ${ }^{+}$. Note the absence of $I_{\text {Trans-LTS }}$ in the presence of NMDG ${ }^{+}$.

isolate this current from the other two CabPK-activated inward currents, and these other currents appeared to contribute substantially to the fast transient peak. In this context, it is noteworthy that $I_{\mathrm{MI}}$ reaches its peak current level relatively quickly in response to a depolarizing voltage step (Fig. 5A). We did not further characterize $I_{\text {Trans-LTF }}$, insofar as it was not necessary for the ability of CabPK to enable gastric mill rhythm generation (see below).

$I_{\text {Trans-LTS }}$ exhibited a longer time to peak than $I_{\text {Trans-LTF }}$ (time to peak at $-45 \mathrm{mV}: 633 \pm 48 \mathrm{~ms}, n=9, p<0.01)$ as well as a larger peak amplitude $(-5.3 \pm 0.6 \mathrm{nA}$, measured at $-15 \pm 3.1$ $\mathrm{mV} ; n=9$; Fig. $3 B, C$ ). It exhibited a voltage threshold of approximately $-55 \mathrm{mV}$ (range, -60 to $-50 \mathrm{mV} ; n=9$ ), and its amplitude increased with depolarization up to approximately $-30 \mathrm{mV}$ $(n=9$; Fig. $3 C)$. Note that this reported peak amplitude value includes the CabPK-activated $I_{\mathrm{MI}}$ amplitude and, at the more depolarized steps, likely also includes the aforementioned voltage- and time-dependent outward current. The unusually 
shallow slope of the $I-V$ curve between -40 and $-10 \mathrm{mV}$ likely results, at least partly, from the contribution of this outward current (Fig. $3 C$ ). The $I_{\text {Trans-LTS }}$ voltage threshold and time to peak suggested that this current was likely to be activated during the CabPK-gastric mill rhythm, during which time the LG neuron membrane potential exhibits rhythmic oscillations between approximately -70 and $-40 \mathrm{mV}$ (Saideman et al., 2007b). We could not measure the full time course of the $I_{\text {Trans-LTS }}$ decay to the baseline because it merged into the sustained inward current, which persisted for the remainder of each step (Figs. 3B, 4A). $I_{\text {Trans-LTS }}$ exhibited a reversal potential that was more depolarized than $0 \mathrm{mV}\left(n=16\right.$ each; Fig. $\left.3 C, I_{\text {Trans-LTS }}\right)$, suggesting that it is primarily carried by ions with positive equilibrium potentials (e.g., $\mathrm{Ca}^{2+}$ and/or $\mathrm{Na}^{+}$). Both of the CabPK-activated transient currents were clearly distinct from $I_{\mathrm{MI}}$, because $I_{\mathrm{MI}}$ exhibits no time-dependent decrease in amplitude (Fig. 5; Golowasch and Marder, 1992).

$I_{\text {Trans-LTS }}$ not only exhibited the property of inactivation, but it also exhibited deinactivation. This deinactivation was sensitive to the time and voltage range that occurs in the LG neuron during the CabPK-gastric mill rhythm. We therefore characterized this property by varying parameters of the voltage step protocol in voltage-clamp experiments. To determine the time dependence of this property, we maintained LG at a holding potential of -40 $\mathrm{mV}$ and systematically hyperpolarized it to $-80 \mathrm{mV}$ for a range of durations ( 1 to $13 \mathrm{~s}$ ), stepping the voltage back to $-40 \mathrm{mV}$ after each hyperpolarization. We then measured the maximum amplitude of the slow transient inward current after the return to -40 $\mathrm{mV}$ (Fig. 4). These data were then fit with a sigmoid curve (Igor Pro), from which two parameters were identified, including the midpoint and slope. There was a relatively long time dependence for $I_{\text {Trans-LTS }}$ deinactivation (midpoint, $7.4 \pm 0.4 \mathrm{~s}$; slope, $2.4 \pm$ $0.2 \mathrm{~s}^{-1} ; n=3$; Fig. $4 B$ ).

We determined the voltage dependence of the $I_{\text {Trans-LTS }}$ deinactivation by varying the prestep voltage across a range $(-75$ to $-50 \mathrm{mV}$ ) of membrane potentials, while maintaining the prestep duration ( $8 \mathrm{~s})$ and subsequent step potential $(-45 \mathrm{mV}$; midpoint, $-60.9 \pm 2 \mathrm{mV}$; slope, $\left.-5.3 \pm 0.5 \mathrm{mV}^{-1}, n=3\right)$. This midpoint value was well within the normal LG neuron membrane potential range $(-55$ to $-70 \mathrm{mV})$ during the gastric mill retraction phase (Saideman et al., 2007b). Thus, based on the time and voltage dependence of this transient current in LG, and the LG neuron membrane potential trajectories during the gastric mill rhythm (Saideman et al., 2007a,b; this study), it likely exhibits considerable inactivation during the course of each gastric mill protraction phase and deinactivation during the retractor phase. These correlations support the hypothesis that the CabPK-activated slow transient inward current helps enable the LG neuron to generate a periodic burst during a pyloric-timed membrane potential depolarization produced by the combination of $I_{\mathrm{MI}}$ and the periodic (AB-mediated) removal of synaptic inhibition from Int1 (see below).

Both CabPK-activated transient inward currents were extracellular $\mathrm{Ca}^{2+}$ dependent. Replacing most of the extracellular $\mathrm{Ca}^{2+}$ with $\mathrm{Mn}^{2+}$, a Ca ${ }^{2+}$ channel antagonist (Turrigiano et al., 1996), consistently resulted in no measureable fast $(n=4)$ or slow transient inward currents (peak $I_{\text {Trans-LTS }}$ at $-50 \mathrm{mV}$ : CabPK alone, $-1.6 \pm 0.1 \mathrm{nA}$; CabPK with $0.1 \mathrm{mM} \mathrm{Ca}^{2+} / 10.9 \mathrm{~mm}$ $\mathrm{Mn}^{2+}, 0 \pm 0 \mathrm{nA}$; CabPK post-reduced $\mathrm{Ca}^{2+},-0.8 \pm 0.02 \mathrm{nA}$; $n=4$; Fig. $5 A$ ). This manipulation had only a moderate effect on $I_{\mathrm{MI}}$ (Fig. 5A), due to the divalent cation sensitivity of this current being approximately equivalent for $\mathrm{Ca}^{2+}$ and $\mathrm{Mn}^{2+}$ (Golowasch and Marder, 1992). In addition, replacing extracellular $\mathrm{Na}^{+}$with

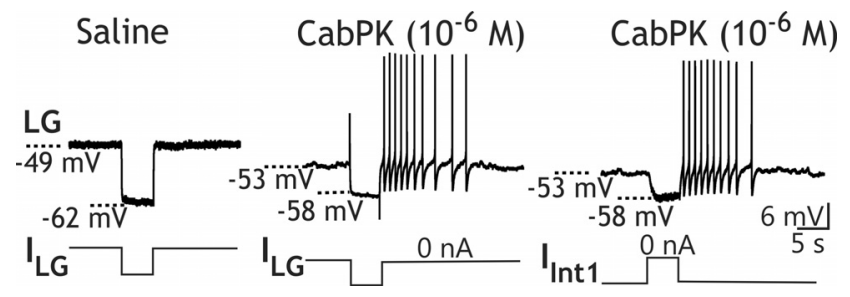

Figure 6. CabPK enables the LG neuron to generate PIR bursts. Left, During saline superfusion, the $L G$ neuron membrane potential was held depolarized by constant depolarizing current injection, during which it was hyperpolarized for $5 \mathrm{~s}$ by current injection $(-1 \mathrm{nA})$. After the hyperpolarization, the $L G$ neuron membrane potential returned directly to its original, depolarized baseline. Middle, In the same LG neuron recording, during CabPK superfusion, a hyperpolarizing current injection (amplitude, $-1 \mathrm{nA}$; duration, $5 \mathrm{~s}$ ) from the CabPK-mediated depolarized baseline was followed by a PIR burst (duration, $8.5 \mathrm{~s}$ ). Note that the last two spikes were excluded from the PIR duration due to their large interspike intervals. Right, In the same preparation, during CabPK application, Int1 activity was suppressed by constant hyperpolarizing current injection and then released from hyperpolarization to fire action potentials for $5 \mathrm{~s}$. This Int1 activity inhibited LG, causing a hyperpolarization comparable to that in the middle trace. When Int 1 activity was again suppressed, $\mathrm{LG}$ generated a PIR burst (duration: $8.9 \mathrm{~s}$ ). The last spike was excluded from the PIR burst duration due to the large interspike interval.

a nonpermeant ionic species $\left(\mathrm{NMDG}^{+}\right)$also consistently resulted in no measureable $I_{\text {Trans-LTS }}$ (maximum amplitude: control, $-3.8 \pm 0.9 \mathrm{nA}$; NMDG saline, $0 \pm 0 \mathrm{nA}, n=7$; Fig. $5 B)$. In contrast, in all four of these $\mathrm{NMDG}^{+}$experiments where there was a discernible $I_{\text {Trans-LTF }}$ peak in the control recordings, this peak persisted in the $\mathrm{NMDG}^{+}$condition (data not shown). In the three experiments where there was no distinguishable $I_{\text {Trans-LTF }}$ peak in the control recording, it was not possible to determine whether it was influenced by the $\mathrm{NMDG}^{+}$substitution (Fig. $5 B$ ).

In sum, these results suggested that $I_{\text {Trans-LTS }}$ is permeable to both $\mathrm{Na}^{+}$and $\mathrm{Ca}^{2+}$, is a $\mathrm{Ca}^{2+}$-sensitive $I_{\mathrm{Na}}$, or is an $I_{\mathrm{CAN}}$, although we also did not rule out the possibility that $\mathrm{NMDG}^{+}$acts instead as an inhibitor of this current. $I_{\mathrm{CAN}}$ was identified previously in C. borealis, both in the stomatogastric nervous system and cardiac ganglion, where it exhibited a reversal potential $\left(V_{\text {rev }}\right)$ of approximately $-30 \mathrm{mV}$ and was insensitive to changes in extracellular $\mathrm{Na}^{+}$but sensitive to caffeine application $\left(10^{-2} \mathrm{M}\right)$, which stimulates intracellular $\mathrm{Ca}^{2+}$ release, and the $I_{\text {CAN }}$ antagonist FFA $\left(10^{-5} \mathrm{M}\right.$; Zhang et al., 1995; Kadiri et al., 2011; Ransdell et al., 2013). However, bath-applied FFA did not alter any of the CabPK-activated currents $(n=2)$. Insofar as $I_{\text {Trans-LTS }}$ exhibited a $V_{\text {rev }}>0 \mathrm{mV}$, sensitivity to extracellular $\mathrm{Na}^{+}$and insensitivity to FFA, it was not likely to be an $I_{\mathrm{CAN}}$.

\section{CabPK-activated transient inward currents enable postinhibitory rebound in the LG neuron}

To determine how the CabPK-activated currents might contribute to LG neuron burst generation during the CabPK-gastric mill rhythm, we examined intrinsic properties in LG. Specifically, we found that brief hyperpolarizing current injections into LG were followed by passive responses during saline superfusion but elicited a PIR burst during CabPK superfusion. Under control conditions (saline), we depolarized LG to a membrane potential that was comparable to its CabPK-mediated baseline potential (approximately $-50 \mathrm{mV}$ ) using either direct depolarizing current injection or DClamp $I_{\mathrm{MI}}$ injection. Despite this depolarized baseline potential, following a period of hyperpolarization there was no evidence of PIR ( $n>10$; Fig. 6). In contrast to its passive response during saline superfusion, LG neuron hyperpolarization from its CabPK-mediated depolarized resting potential was consistently followed by a PIR burst (Fig. 6). Specifically, during 
CabPK superfusion, injecting a modest hyperpolarizing current ( $-1 \mathrm{nA})$ into LG, which caused a 5-10 $\mathrm{mV}$ hyperpolarization, consistently elicited a PIR burst when the current injection was terminated (PIR burst: duration, $5.14 \pm 0.2 \mathrm{~s}$; number of spikes: $8.22 \pm 0.2 ; n=9)$. We used hyperpolarizing durations ( $5 \mathrm{~s}$ ) and amplitudes that were similar to those experienced by LG during the gastric mill retraction phase. The trough of the subthreshold LG neuron oscillations during retraction ranged from -55 to $-65 \mathrm{mV}$ across experiments (Saideman et al., 2007a,b).

The LG neuron also readily exhibited PIR bursts during CabPK application after an episode of synaptic inhibition from Int1 ( $n=5$; Fig. 6). To establish Int1 mediated PIR in LG, Int1 activity was suppressed via hyperpolarizing current injection and periodically released from this hyperpolarization to fire action potentials for $5 \mathrm{~s}$, comparable to its active period during the gastric mill rhythm. The resulting inhibition in LG caused it to hyperpolarize by $10.2 \pm$ $1.5 \mathrm{mV}(n=5)$, comparable to its response to Int1 during gastric mill retraction (Saideman et al., 2007a,b). At the end of each inhibitory episode, LG generated a PIR burst comparable to those resulting from hyperpolarizing current injection (PIR burst duration, $5.3 \pm 1 \mathrm{~s}$; number of spikes, $8.2 \pm$ $0.6 ; n=5 ; p>0.5$ for both parameters).

PIR bursts are often driven at least partly by the hyperpolarization-activated inward current $\left(I_{\mathrm{h}}\right.$; McCormick and Bal, 1997; Sekirnjak and du Lac, 2002; Robinson and Siegelbaum, 2003; Sangrey and Jaeger, 2010; Engbers et al., 2011; Felix et al., 2011). However, $I_{\mathrm{h}}$ did not appear to contribute to the CabPK-enabled PIR bursts in LG insofar as neither hyperpolarizing current injection nor synaptic inhibition from Int 1 revealed any evidence of a depolarizing sag potential during saline superfusion $(n>10)$ or CabPK application ( $n=18 / 20$; Figs. 6 , 7). Similarly, there was no evidence in our voltage-clamp experiments for a sag current $(n>10)$. However, the CabPKactivated $I_{\text {Trans-LTS }}$, with its voltage- and time-dependent properties of inactivation and deinactivation, was a candidate for the ionic current underlying PIR burst generation.

We first assessed the contribution of this CabPK-activated transient inward current to PIR in LG by simplifying the preparation with $\operatorname{TTX}\left(10^{-7} \mathrm{M}\right)$ saline to silence all neurons. There was no evidence for a PIR response after LG was hyperpolarized in TTX saline either from its resting potential $(-59.1 \pm 1.1 \mathrm{mV} ; n=$ 18 ) or from a depolarized membrane potential $(-47.4 \pm 1 \mathrm{mV}$; $n=13$; Fig. $7 A)$. In contrast, during CabPK $\left(10^{-6} \mathrm{M}\right)$ application
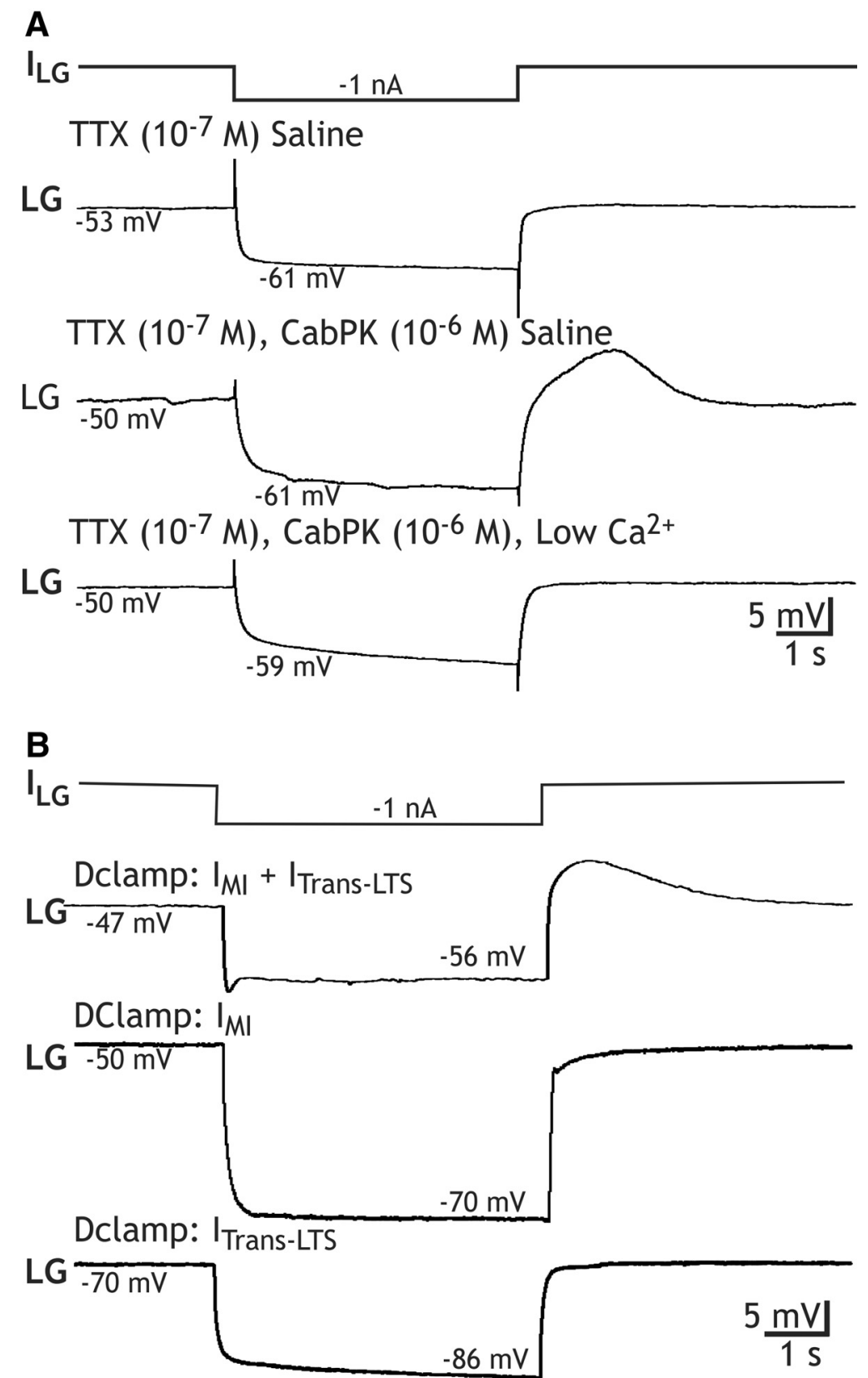

Figure 7. PIR in the $L G$ neuron persists in the presence of TTX during either CabPK application or dynamic-clamp coinjection of artificial $I_{M}$ plus $I_{\text {Trans-LIS }} \cdot A$, Top, In the presence of TTX saline, a 5 s hyperpolarization in the $L G$ neuron from a depolarized baseline was followed by a direct return to the depolarized baseline. Middle, During CabPK superfusion in TTX saline, a comparable $5 \mathrm{~s}$ hyperpolarization in $L G$ was followed by PIR, albeit without action potentials. Here, the depolarized baseline resulted from the influence of CabPK. Bottom, CabPK superfusion in TTX saline containing $0.1 \times$ normal $\mathrm{Ca}^{2+}$, substituted with equimolar $\mathrm{Mn}^{2+}$, did not enable PIR after the same hyperpolarizing step as above. All recordings were from the same LG neuron. $\boldsymbol{B}$, Top, Dynamic clamp injection of artificial $I_{M I}\left(g_{M 1} 80 \mathrm{nS}\right)$ plus $I_{\text {Trans-LTS }}\left(g_{\text {Trans-LTS, }} 100 \mathrm{nS}\right)$ in TTX saline enabled PIR in the LG neuron after a hyperpolarizing step. The initial dip in the $L G$ neuron membrane potential occurred in all $(n=4)$ of these responses to hyperpolarizing current injection during the dynamic-clamp coinjections but not during the individual injections (e.g., see below). Middle, bottom, In contrast, separate dynamic-clamp injection of $I_{\text {MI }}$ (middle) or $I_{\text {Trans-LTS }}$ (bottom) in TTX saline did not enable PIR in the LG neuron. Note that, in the bottom recording without artificial $I_{M \prime}$, the LG neuron resting potential is not depolarized.

under this condition, LG again exhibited a maintained depolarization $(-48.4 \pm 1.2 \mathrm{mV}, n=18)$ from which it displayed PIR in response to hyperpolarizing pulses $(-1 \mathrm{nA})$, albeit without associated action potentials $(n=18$; Fig. $7 A)$. Thus, this CabPKmediated PIR did not require activation of TTX-sensitive $I_{\mathrm{Na}}$. However, these PIR events were briefer than the PIR bursts that occurred during normal CabPK saline $(2.0 \pm 0.1 \mathrm{~s} ; n=18$; $p<$ 


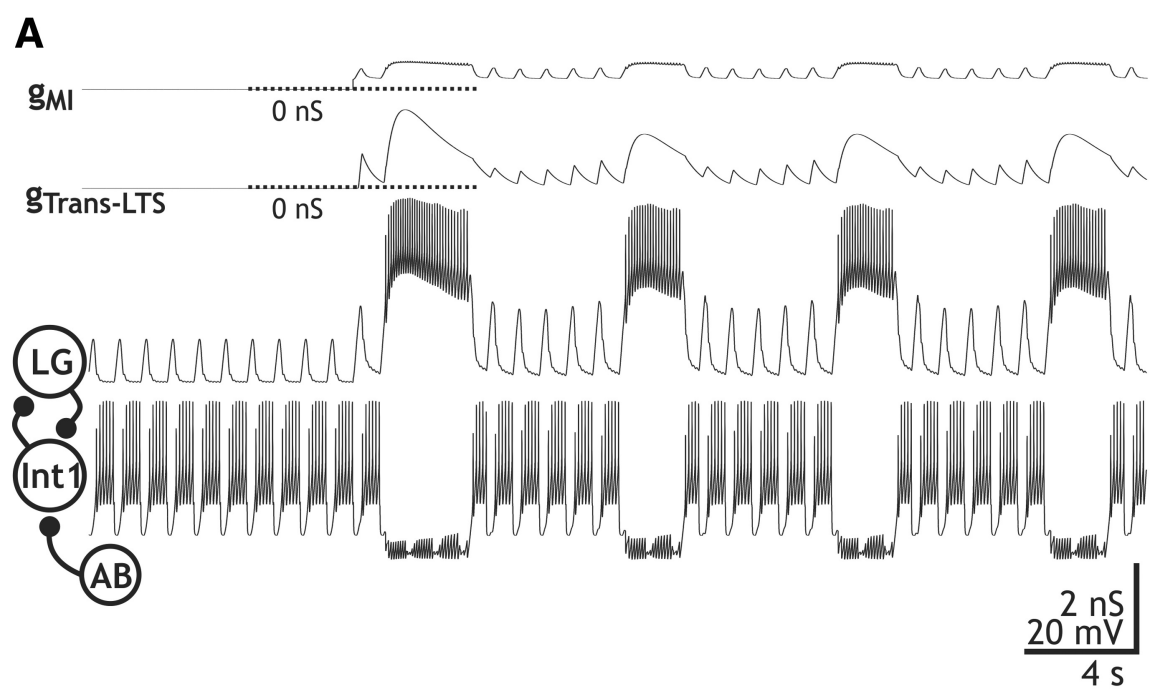

B

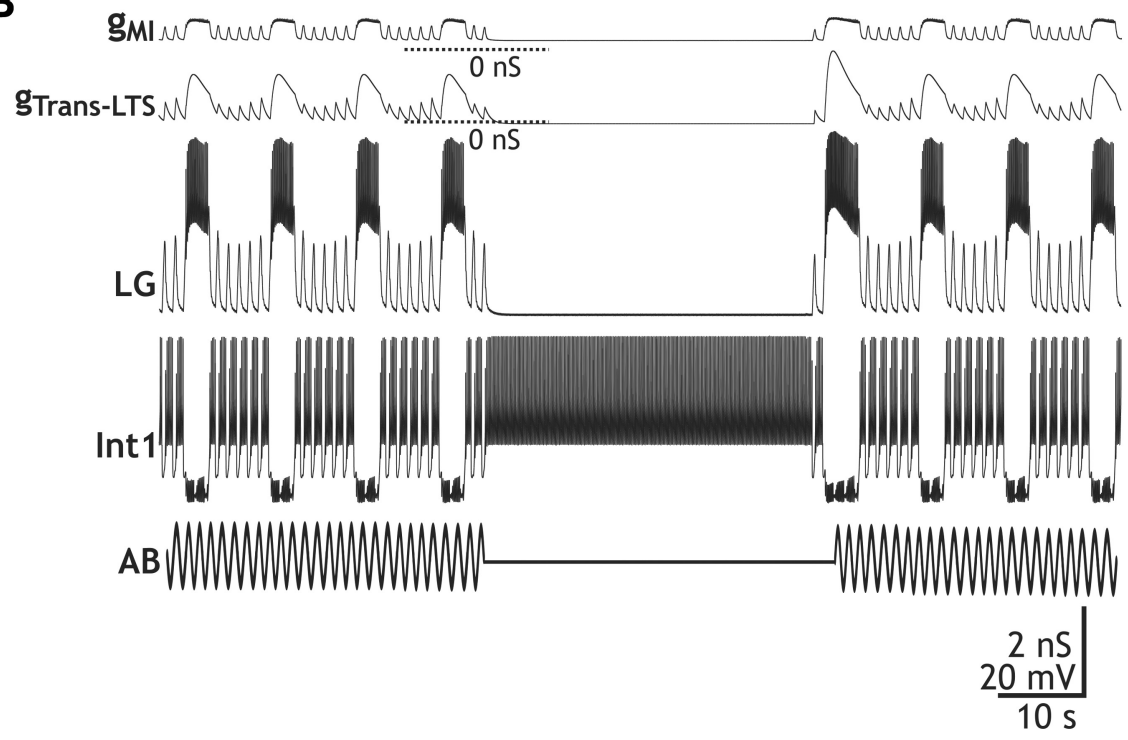

Figure 8. CabPK-like gastric mill rhythm generation output from a computational model that includes models of the LG, Int1, and $A B$ neurons. $A$, Output of the computational model showing the rhythmic alternating bursting in $L G$ and Int 1 neurons that commences upon activating the CabPK-activated conductances $\left(g_{\text {Trans-LTS }} ; g_{M \mathrm{II}}\right)$. The AB neuron influence is evident in the fast rhythmic Int1 hyperpolarizations and the associated subthreshold depolarizations (removal of Int1 inhibition) in the LG neuron. Note that the CabPK-activated conductances all track the membrane potential changes in LG, while $g_{\text {Trans-ITs }}$ also exhibits timedependent processes, including inactivation during the $L G$ neuron burst and an increasing level of activation (due to deinactivation) during the $L G$ neuron interburst. Most hyperpolarized membrane potentials: Int1, $-60.5 \mathrm{mV} ; \mathrm{LG},-58.4 \mathrm{mV}$. Filled circle, inhibition. $\boldsymbol{B}$, Suppressing the $A B$ inhibition of Int 1 terminates the ongoing gastric mill rhythm in the computational model of the CabPK-gastric mill rhythm generator, as occurs in the biological system (Bartos et al., 1999). When the AB neuron activity was temporarily terminated (horizontal line, middle of the $A B$ trace), Int 1 activity changed from a fast rhythmic pattern to tonic firing, while the LG neuron membrane potential exhibited a steady hyperpolarized potential. Note that, due to the hyperpolarized LG neuron membrane potential, $g_{\text {Trans-LTS }}$ was at $0 \mathrm{nS}$ while $g_{\mathrm{MI}}$ was maintained at a reduced level. Most hyperpolarized membrane potentials: Int1, $-47.7 \mathrm{mV} ; \mathrm{LG},-58.4 \mathrm{mV}$.

0.01 ), suggesting that a TTX-sensitive $I_{\mathrm{Na}}$ might prolong this response.

We tested the role in PIR generation played by the $I_{\mathrm{MI}^{-}}$ associated depolarization in the LG neuron during CabPK application. To this end, during CabPK application in TTX saline, we injected constant amplitude hyperpolarizing current to return the LG neuron membrane potential to its pre-CabPK resting potential. That is, we eliminated the effect of CabPK-activated $I_{\mathrm{MI}}$, which underlies the steady LG neuron depolarization (see above). From that resting potential, we again injected hyperpolarizing current pulses as above. Doing so reduced the PIR amplitude to $\sim 25 \%$ of the control amplitude (control: $V_{\mathrm{m}}=-51.1 \pm 2 \mathrm{mV}$; PIR amplitude, $7.2 \pm 0.4 \mathrm{mV}$; hyperpolarized: $V_{\mathrm{m}}=$ $-59.1 \pm 2 \mathrm{mV} ;$ PIR amplitude, $1.8 \pm 0.7$ $\mathrm{mV} ; n=7 ; p<0.01$ ), supporting the hypothesis that the $I_{\mathrm{MI}}$-mediated depolarization in LG neuron strengthens PIR generation mediated by activation of the transient inward current (see below).

We next assessed whether this CabPKenabled PIR event in TTX saline was $\mathrm{Ca}^{2+}$-sensitive by applying CabPK after replacing most of the $\mathrm{Ca}^{2+}(0.1 \times$ normal $)$ in the saline with an equimolar concentration of $\mathrm{Mn}^{2+}$. Under these conditions, the PIR amplitude was reversibly reduced (precontrol, $7.5 \pm 0.7 \mathrm{mV}$; reduced $\mathrm{Ca}^{2+} /$ added $\mathrm{Mn}^{2+}$ saline, $0.4 \pm 0.1 \mathrm{mV} ; n=6, p<0.01$; Fig. $7 A$ ). These results suggested that the CabPK-elicited PIR was mediated by activation of the CabPK-activated low-threshold inward currents.

We tested the hypothesis that the CabPK-activated $I_{\text {MI }}$ plus $I_{\text {Trans-LTs }}$ was sufficient to enable PIR in LG. To this end, in TTX saline, we injected into LG a dynamic-clamp version of these two currents. This manipulation did indeed enable LG to express PIR in response to the same hyperpolarizing current injections used in the presence of CabPK (PIR amplitude, $8.7 \pm 1.5 \mathrm{mV}$; PIR duration, $4.5 \pm 0.03 \mathrm{~s} ; n=4$; Fig. $7 B$ ). Performing the same manipulation using only the DClamp version of $I_{\mathrm{MI}}$ or $I_{\text {Trans-LTS }}$ did not elicit PIR $(n=4$; Fig. $7 B)$. The absence of PIR during the latter manipulation likely resulted from the absence of a depolarized LG neuron resting potential, which in turn limited the activation of the transient inward current after the hyperpolarizing current injection. Thus, the ability of CabPK to enable the LG neuron to generate PIR bursts apparently results from its coactivation of $I_{\mathrm{MI}}$, to depolarize LG, and $I_{\text {Trans-LTS }}$, to provide the drive for the PIR burst.

\section{CabPK-activated inward currents in the} LG neuron are necessary for gastric mill rhythm generation

We tested the ability of the identified CabPK-activated inward currents in the LG neuron to enable gastric mill rhythm generation in a computational model, after which we tested the predictions of the model in the biological system. We developed a computational model of the CabPK-gastric mill rhythm generator (LG, Int 1, and AB neurons) in which LG contained CabPK-activated $I_{\mathrm{MI}}$ plus $I_{\text {Trans-LTS }}$ (see Materials and Methods; Table 2). This model was based on one of three previously published models (CabPK Mechanism 1) that were focused on distinct candidate mechanisms for CabPKmediated gastric mill rhythm generation (Kintos et al., 2008). As shown in Figure $8 A$, our model produced gastric mill rhythm-like alternating bursting in LG and Int 1 . The model rhythm exhibited 
a cycle period $\left(10.8 \pm 2 \times 10^{-4} \mathrm{~s}\right)$, LG neuron burst duration $\left(2.75 \pm 1 \times 10^{-4}\right.$ s), and LG neuron interburst duration $\left(8.09 \pm 1 \times 10^{-4} \mathrm{~s}\right)$ similar to the biological CabPK-gastric mill rhythm (cycle period, $11.96 \pm 1.1 \mathrm{~s}$; LG neuron burst duration, CabPK, $3.2 \pm 0.3 \mathrm{~s}$; LG neuron interburst duration, $8.8 \pm 1.1 \mathrm{~s} ; n=12$ ). Additionally, the average protraction and retraction duty cycles (DCs; fraction of the cycle) were comparable (protraction DC: model, 0.25 ; biological, 0.27 ; retraction DC: model, 0.75; biological, 0.73). Note that, during the model rhythm, $g_{\mathrm{MI}}$ and $g_{\text {Trans-LTS }}$ followed the LG neuron voltage trajectory (Fig. $8 A$ ), insofar as their activation was voltage dependent. The deinactivation $(h)$ state of $g_{\text {Trans-LTs }}$ also tracked the LG neuron membrane potential, rising during the retractor phase when LG was rhythmically hyperpolarized by Int 1 inhibition.

The model CabPK-gastric mill rhythm was also comparable to the biological rhythm in that it was suppressed by eliminating the $A B$ inhibition of Int 1 (Fig. $8 B$ ). In the biological system, this manipulation terminates the CabPK-gastric mill rhythm, but not the MCN1-gastric mill rhythm (Bartos et al., 1999; Saideman et al., 2007b). As discussed above, during the gastric mill rhythm each fast rhythmic depolarization (disinhibition) in the LG neuron results from the fast rhythmic $\mathrm{AB}$ inhibition of Int 1 unmasking the depolarizing drive in LG due to CabPK-activated $I_{\mathrm{MI}}$ and $I_{\text {Trans-LTs }}$. When the $A B$ inhibition of Intl is suppressed, Int1 fires tonically (Bartos et al., 1999). Selectively silencing AB in the model CabPK-gastric mill rhythm-generating circuit did cause Int 1 to fire tonically and eliminated the rhythmic disinhibitions in LG that normally provide the trigger for each LG burst (Fig. $8 B$ ). Silencing AB also resulted in the Int1 inhibition dominating the LG neuron membrane potential, keeping LG too hyperpolarized to activate sufficient $g_{\mathrm{MI}}$ and $g_{\text {Trans-LTS }}$ (Fig. $8 B)$.

Removing either $I_{\mathrm{MI}}$ or $I_{\text {Trans-LTS }}$ from the model LG neuron eliminated gastric mill rhythm generation, supporting the hypothesis that these currents are necessary for this process (Fig. $9 A, B)$. When either the model $g_{\mathrm{MI}}$ or $g_{\text {Trans-LTS }}$ was removed (i.e., set to $0 \mathrm{nS}$ ), the pyloric-timed LG neuron membrane potential oscillation peak amplitude was reduced, preventing LG from reaching action potential threshold and terminating the model gastric mill rhythm (Fig. 9A,B). The peak amplitude of these subthreshold oscillations in LG was larger after selectively removing $g_{\text {Trans-LTS }}(12.92 \pm 3.4 \mathrm{mV}$; Fig. $9 B)$ than after selectively removing $g_{\mathrm{MI}}(7.32 \pm 1.6 \mathrm{mV}$; Fig. $9 A)$. This result was not surprising, given that with $I_{\mathrm{MI}}$ absent the subthreshold oscillations peaked at a membrane potential close to threshold for $I_{\text {Trans-LTS }}$ activation (Fig. 3).

We evaluated the predictions of the computational model in the biological system using dynamic-clamp current injections into LG. We tested the necessity of $I_{\mathrm{MI}}$ and $I_{\text {Trans-LTS }}$ for CabPK- gastric mill rhythm generation by selectively nullifying each one during the CabPK-gastric mill rhythm. We performed each such manipulation by using the dynamic-clamp to provide negative versions of each conductance, enabling injection of artificial $I_{\mathrm{MI}}$ or $I_{\text {Trans-LTS }}$ that was approximately equal in amplitude and opposite in sign to the CabPK-activated version of that same current (DeLong and Nusbaum, 2010).

As was the case during the comparable manipulations in our computational model (Fig. 9), moderate levels of negative conductance injection (range, 50-200 pS) of either $g_{\mathrm{MI}}$ or $g_{\text {Trans-LTS }}$ caused only a modest reduction in the peak depolarization of the pyloric-timed oscillations in LG, but it was sufficient to arrest LG neuron bursting and terminate the ongoing gastric mill rhythm for the duration of the manipulation ( $g_{\mathrm{MI}}, n=7 ; g_{\text {Trans-LTS }}, n=5$; Fig. 10). In most experiments, we tested a twofold range of negative conductance values and found that they all effectively suppressed the ongoing rhythm. These manipulations not only suppressed rhythmic bursting in LG, but they eliminated the entire gastric mill rhythm. For example, the combined pyloric- and gastric mill-timed patterns of the IC (inferior cardiac) and VD neurons were replaced by an exclusively py- 
A $\operatorname{CabPK}\left(10^{-6} \mathrm{M}\right)$

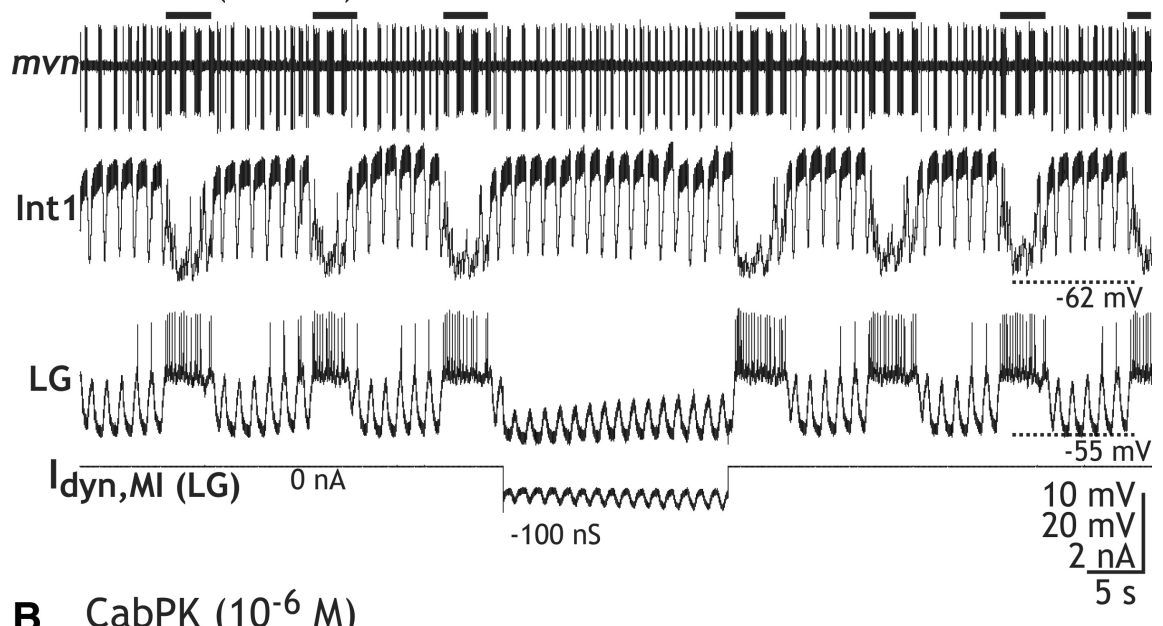

B $\operatorname{CabPK}\left(10^{-6} \mathrm{M}\right)$

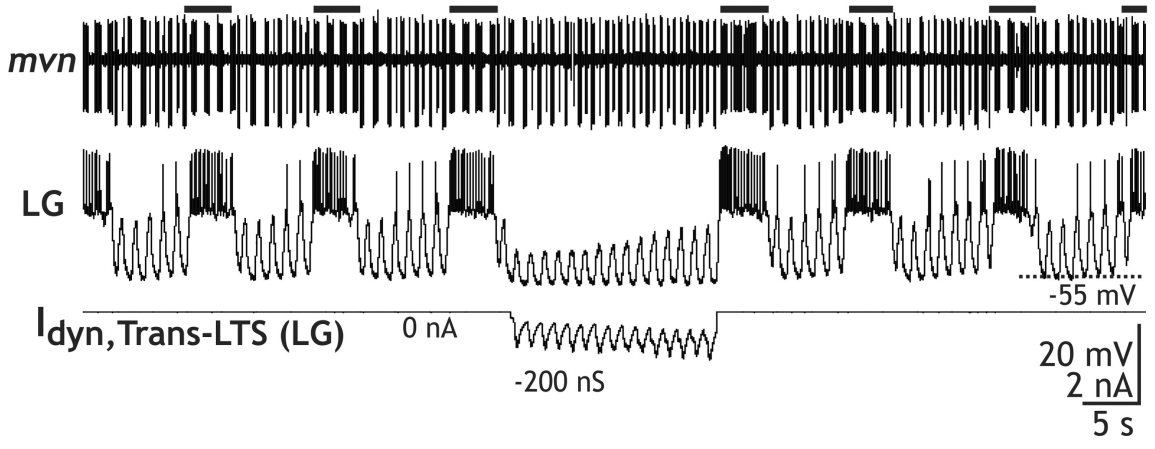

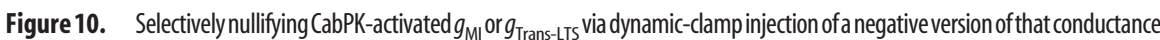
suppressed an ongoing CabPK- gastric mill rhythm. $\boldsymbol{A}, \boldsymbol{B}$, Injecting into the $L G$ neuron a negative version of $g_{\mathrm{MI}}\left[I_{\text {dyn,MI (LG) }}\right](\boldsymbol{A})$ or $g_{\text {Trans-LTS }}$ $\left[I_{\text {Trans-LTS (LG) }}\right](\boldsymbol{B})$ reduced the amplitude of the LG neuron subthreshold oscillations and prevented its bursting. During this time, Int1 activity remained pyloric timed, while the IC ( $m v n$ : small unit) and VD ( $m v n$ : large unit) neuron activity switched from exhibiting gastric mill-and pyloric-timed bursting to exclusively pyloric-timed activity. Black bars, Gastric mill protraction phase-timed IC neuron bursting. Both panels are from the same preparation.

loric rhythm-timed pattern ( $n=3$ of 3 preparations; Fig. 10 ). The gastric mill rhythm consistently resumed when the dynamic-clamp injection was terminated (Fig. 10). Thus, both CabPK-activated $I_{\mathrm{MI}}$ and $I_{\text {Trans-LTS }}$ in the LG neuron were necessary for gastric mill rhythm generation.

\section{CabPK-activated inward currents in the LG neuron are sufficient for gastric mill rhythm generation}

To determine whether the CabPK-activated $I_{\mathrm{MI}}$ and $I_{\text {Trans-LTS }}$ in the LG neuron were sufficient for gastric mill rhythm generation, as was the case in the computational model, we first combined a dynamic-clamp injection of $I_{\text {Trans-LTs }}$ into the LG neuron with bath application of the muscarinic agonist OXO $\left(10^{-5} \mathrm{M}\right)$, an $I_{\mathrm{MI}}$ activator in pyloric circuit neurons (Swensen and Marder, 2000, 2001). We established via voltage-clamp experiments that OXO consistently activated $I_{\mathrm{MI}}$ in the LG neuron as well $(n=5)$. In parallel current-clamp experiments in which hyperpolarizing current steps were injected into LG during OXO superfusion, PIR was not elicited $(n=5)$. The OXO-activated $I_{\mathrm{MI}}$ characteristics in the LG neuron were comparable to those resulting from CabPK and CCAP application (e.g., a relatively steady and smallamplitude inward current at potentials more hyperpolarized than $-60 \mathrm{mV}(-0.3 \pm 0.04 \mathrm{nA}, n=5)$, and a similar $V_{\mathrm{m}}$ for peak amplitude $(-7.6 \pm 3.8 \mathrm{mV}, n=5)$, although the peak amplitude was lower relative to these peptides $(-1.2 \pm 0.27 \mathrm{nA}, n=5$;
DeLong et al., 2009a; this paper). OXO $\left(10^{-5} \mathrm{M}\right)$ superfusion consistently increased the amplitude of the subthreshold pyloric-timed oscillations in LG by $5-10$ $\mathrm{mV}(n=10)$, by selectively increasing their depolarized peak $V_{\mathrm{m}}$ (peak $V_{\mathrm{m}}$ : control, $-54.2 \pm 1.7 \mathrm{mV}$; OXO, $45.8 \pm 2 \mathrm{mV}$; $n=10, p<0.01$; trough $V_{\mathrm{m}}$ : control, $-55.2 \pm 1.8 \mathrm{mV} ; \mathrm{OXO},-55.7 \pm 1.9 ; n=$ $10, p=0.13$ ), without activating LG neuron bursting (Fig. 11A). This result was comparable to that occurring during CabPK applications with dynamicclamp nullification of $I_{\text {Trans-LTS }}$, when the only CabPK-activated inward current influencing the LG neuron membrane potential was $I_{\mathrm{MI}}$ (Fig. 10B).

During OXO $\left(10^{-5} \mathrm{M}\right)$ superfusion, injecting $I_{\text {dyn,Trans-LTS }}(50-100 \mathrm{nS}$; peak current, 1-3 nA) enabled LG to generate gastric mill rhythm-like bursting $(n=5$; Fig. $11 B$ ). This rhythmic bursting in LG exhibited characteristics that were similar to those occurring during the CabPKgastric mill rhythm $(n=12)$, such as its intraburst firing frequency $(\mathrm{OXO} /$ DClamp, $3.9 \pm 0.6 \mathrm{~Hz}$; CabPK, $2.7 \pm 0.2$ $\mathrm{Hz} ; p=0.14)$, burst duration $(\mathrm{OXO} /$ DClamp, $2.3 \pm 0.3 \mathrm{~s}$; CabPK, $3.2 \pm 0.3 \mathrm{~s}$; $p=0.17)$, and interburst duration (OXO/ DClamp, $6.4 \pm 0.8 \mathrm{~s}$; CabPK, $8.8 \pm 1.1 \mathrm{~s}$; $p=0.41)$. These results were consistent across a twofold range of dynamic-clamp conductances (e.g., 50-100 nS) within the same experiments.

In the above OXO experiments, it remained possible that rhythm generation resulted in part from additional OXO actions on other neurons (e.g., Int1, AB) necessary for gastric mill rhythm generation. Therefore, to determine whether the CabPKactivated inward currents in LG were likely to be truly sufficient to enable a gastric mill rhythm-like pattern in LG, we coinjected into the biological LG neuron dynamic-clamp versions of $I_{\mathrm{MI}}$, $I_{\text {Trans-LTs }}$ plus the Int1-mediated synaptic inhibition. These experiments were performed in PTX saline to isolate the LG neuron. These dynamic-clamp coinjections consistently elicited gastric mill rhythm-like bursting in LG $(n=4$; Fig. 12$)$. The resulting cycle period $(8.92 \pm 1.7 \mathrm{~s}, p>0.05)$, LG neuron burst duration $(1.83 \pm 0.2 \mathrm{~s}, p>0.05)$, interburst duration (7.09 \pm $0.8 s, p>0.05)$, and the number of spikes per burst (11.38 \pm 0.9 , $p>0.05)$ were all similar to the above-reported values during the CabPK-gastric mill rhythm (Fig. 13). These experiments therefore supported the hypothesis that the CabPK-activated $I_{\mathrm{MI}}$ plus $I_{\text {Trans-LTs }}$ in the LG neuron were sufficient to enable gastric mill rhythm-equivalent alternating bursting in the core gastric mill rhythm generator neurons LG and Int 1 .

\section{Discussion}

We have identified the cellular mechanisms underlying CabPK neuropeptide activation of a rhythmic motor pattern, thereby establishing that these mechanisms are distinct from those by which a previously studied modulatory pathway activates the same motor pattern. Specifically, bath applying CabPK or stim- 
ulating the projection neuron MCN1 configure different gastric mill circuits yet elicit the same gastric mill motor pattern (Fig. 1B; Saideman et al., 2007b). Here we demonstrated that CabPK peptideelicited gastric mill rhythm generation results from its persistent recruitment of at least two voltage-dependent inward currents $\left(I_{\mathrm{MI}}, I_{\text {Trans-LTS }}\right)$ in the rhythm generator neuron LG. These currents conjointly enable LG to rhythmically generate a PIR burst and thereby produce an alternating activity pattern with the rhythm generator neuron Int1, which they then impose on the other gastric mill motor neurons via their synaptic actions (Fig. 1B).

Our computational model and dynamicclamp manipulations support the hypothesis that the CabPK-elicited rhythmic LG neuron bursting results from $I_{\mathrm{MI}}$ depolarizing LG closer to its spike threshold, enabling the voltage- and time-dependent properties of $I_{\text {Trans-LTS }}$ to periodically generate a PIR burst. The projection neuron MCN1 also activates the gastric mill rhythm generator via $I_{\mathrm{MI}}$ activation in LG (DeLong et al., 2009a). However, the MCN1-activated $I_{\mathrm{MI}}$ grows and decays during the gastric mill retractor and protractor phases, respectively, due to continual neuropeptide release from MCN1 during retraction and presynaptic feedback inhibition of that release during protraction. This MCN1-mediated rhythmic buildup of $I_{\mathrm{MI}}$ is sufficiently strong to enable LG to periodically reach spike threshold and generate a selfterminating burst (Coleman et al., 1995; Bartos et al., 1999; DeLong et al., 2009a).

Different processes can potentially enable the same rhythmic motor pattern to be elicited by different modulatory inputs. These processes include convergent activation of the same direct input to a circuit (Di Prisco et al., 2000; Korn and Faber, 2005; Derjean et al., 2010), convergent modulation of the same properties in the same network neurons (Doi and Ramirez, 2010), divergent modulation in network neurons of multiple baseline intrinsic and synaptic conductances, which functionally compensate for one another (MacLean et al., 2003; Prinz et al., 2004a; Goaillard et al., 2009; Grashow et al., 2010; Norris et al., 2011), or configuration of different circuits by activating distinct conductances that enable different intrinsic properties in network neurons (Saideman et al., 2007b; this article). The crab gastric mill system appears to provide the first example of the latter of these mechanisms.

Despite MCN1 and CabPK configuring different rhythmgenerating circuits, at their core both circuits include the reciprocally inhibitory neurons LG and Int 1 . These two neurons establish the protractor and retractor phases, respectively, and
A Normal Saline

Idyn, Trans-LTS (LG) $0 \mathrm{nA}$

OXO $\left(10^{-5} \mathrm{M}\right)$
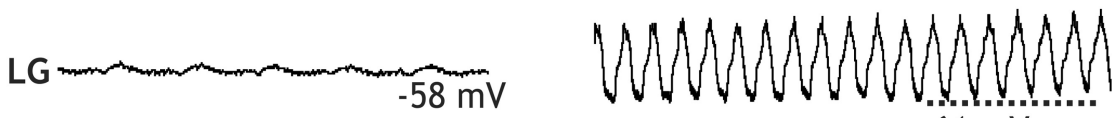

$-61 \mathrm{mV}$

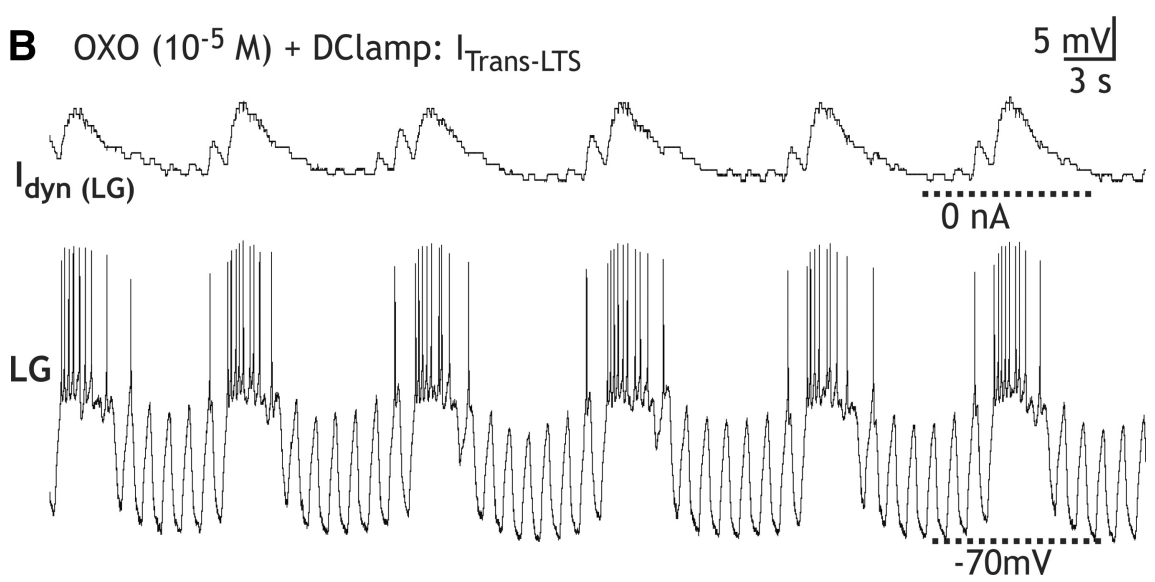

Figure 11. Gastric mill rhythm-equivalent bursting in the $L G$ neuron during application of the $I_{M I}$ activator $0 X 0$ plus dynamic-

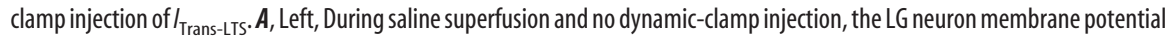
exhibited small-amplitude pyloric-timed oscillations. Right, $0 \times 0$ application increased the amplitude of these oscillations, but they remained subthreshold. These subthreshold oscillations exhibited a more depolarized peak, presumably due to $0 \mathrm{XO}$-activated $I_{\mathrm{Ml}}$ and a more hyperpolarized trough, presumably due to OXO excitation of Int1 (Norris et al., 1994). The oscillation frequency also increased, because OXO also excites the pyloric CPG (Bal et al., 1994). B, Dynamic-clamp injection of $\left.\right|_{\text {Trans-LTS }}\left(g_{\text {Trans-LTS }}, 100 \mathrm{nS}\right)$ during $0 X 0$ superfusion elicited gastric mill-equivalent bursting in the $L G$ neuron.
A PTX $(50 \mu M)$ Saline

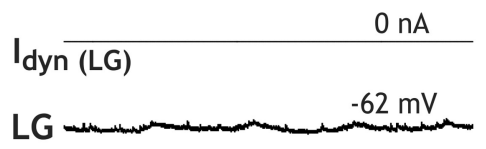

Dclamp: Int1 Synapse $+I_{M I}$

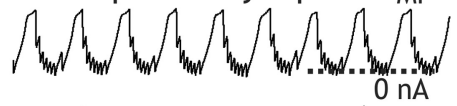

$2 \mathrm{nA}$

B Dclamp: Int1 Synapse $+I_{M I}+I_{\text {Trans-LTS }}$ $\frac{\mathrm{mV}}{1 \mathrm{~s}}$
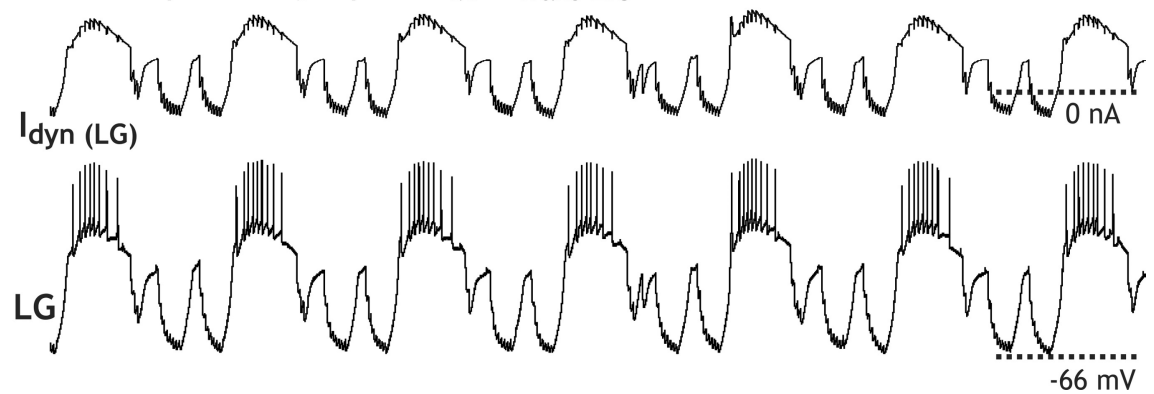

Figure 12. Dynamic-clamp coinjection of all CabPK-gastric mill rhythm generator conductances into the $L G$ neuron elicits gastric mill rhythm-like bursting in the LG neuron. $A$, Left, In PTX saline with no dynamic-clamp injection, the LG neuron maintained a steady resting potential of $-62 \mathrm{mV}$. Right, Dynamic-clamp coinjection of the inhibitory synapse from Int 1 (200 nS) plus CabPK-activated $I_{M I}(80 \mathrm{nS})$ in PTX saline elicited subthreshold, pyloric rhythm-like oscillations in LG. B, Dynamic-clamp coinjection into LG of the rhythmic inhibitory synapse from Int 1 (200 nS), CabPK-activated $I_{\text {MI }}(80 \mathrm{nS})$, plus CabPK-activated $I_{\text {Trans-LTS }}(100 \mathrm{nS})$ in PTX saline elicited gastric mill rhythm-like bursting in LG.

in each case the pivotal rhythm-generating event is LG neuron activation, presumably because Int 1 is spontaneously active (Bartos et al., 1999; Saideman et al., 2007b). However, despite these similarities, and the fact that MCN1 and CabPK each recruit $I_{\mathrm{MI}}$, they enable different active properties in LG. These 


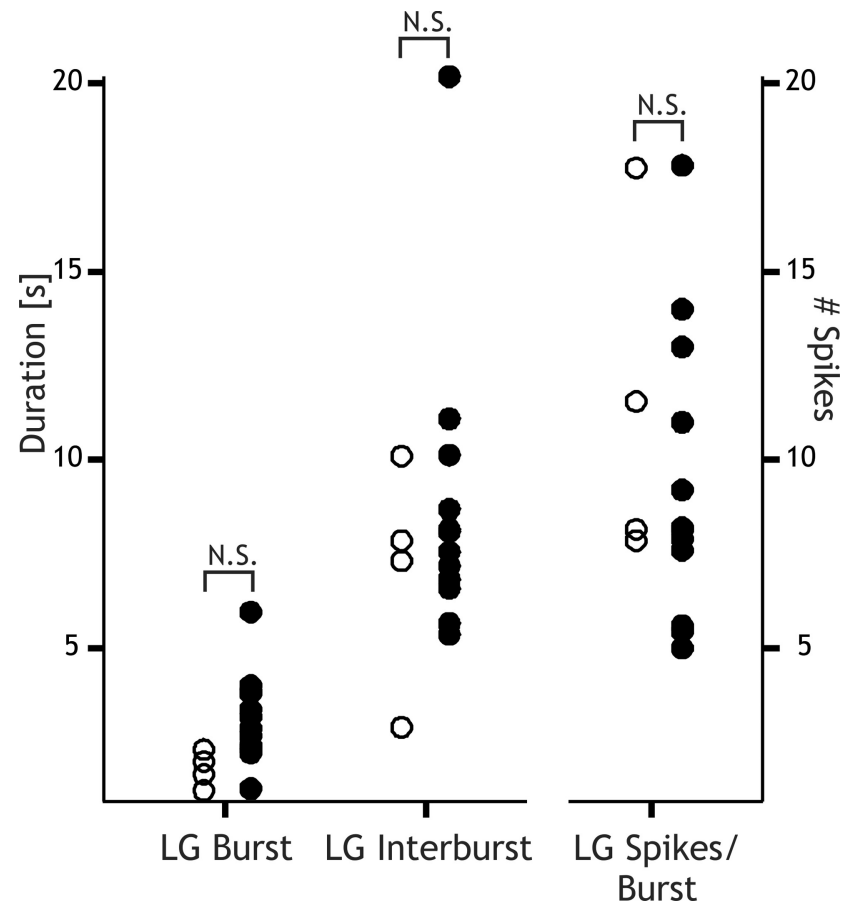

Figure 13. The range of the gastric mill rhythm-related parameters in the LG neuron are comparable during the biological CabPK- gastric mill rhythm and when artificial versions of synaptic inhibition plus the CabPK-activated conductances are coinjected into LG. Distribution of mean values is shown for the $L G$ neuron burst duration, interburst duration, and number of spikes per burst during CabPK- gastric mill rhythms (filled circles: $n=11$ ) and dynamic-clamp-elicited gastric mill rhythm-like bursting in $L G(n=4)$. All error bars (SEM) are smaller than the associated circles. N.S., Not significantly different ( $p>0.05$ ).

different properties result from the distinct temporal dynamics of $I_{\mathrm{MI}}$ activation by these two pathways, the likelihood that MCN1 activates more $I_{\mathrm{MI}}$ than CabPK (see below), and the fact that only CabPK appears to activate $I_{\text {Trans-LTS }}$ (Bartos et al., 1999; DeLong et al., 2009a; this article).

PIR bursts often involve the complementary influence of multiple voltage-dependent inward currents, as during CabPK modulation of the LG neuron (McCormick and Bal, 1997; Sekirnjak and du Lac, 2002; Angstadt et al., 2005; Sangrey and Jaeger, 2010; Wang et al., 2011; Engbers et al., 2011; Felix et al., 2011; Zheng and Raman, 2011). One inward current that often contributes to the initial PIR depolarization is $I_{\mathrm{h}}$ (McCormick and Bal, 1997; Sekirnjak and du Lac, 2002; Robinson and Siegelbaum, 2003; Pirtle et al., 2010; Sangrey and Jaeger, 2010; Engbers et al., 2011; Felix et al., 2011). The $I_{\mathrm{h}}$-mediated rebound depolarization facilitates activation of other voltagedependent inward currents that elicit or strengthen the PIR burst. These latter currents often have time- and voltagedependent properties similar to those of CabPK-activated $I_{\text {Trans-LTS. In many neurons, the additional PIR-generating }}$ current is some type of $I_{\mathrm{Ca}}$ (McCormick and Bal, 1997; Angstadt et al., 2005; Sangrey and Jaeger, 2010; Wang et al., 2011; Engbers et al., 2011; Felix et al., 2011). The role of $I_{\mathrm{h}}$ in PIR bursts is similar to that of CabPK-activated $I_{\mathrm{MI}}$ in LG, which depolarizes the LG neuron after a period of inhibition and enables sufficient $I_{\text {Trans-LTS }}$ activation to generate the PIR burst. Also, similar to the CabPK comodulation of $I_{\mathrm{MI}}$ and $I_{\text {Trans-LTS }}$, the complementary PIR-generating currents in some other systems can be comodulated (Harris-Warrick et al., 1995; Angstadt et al., 2005; Wang et al., 2011).
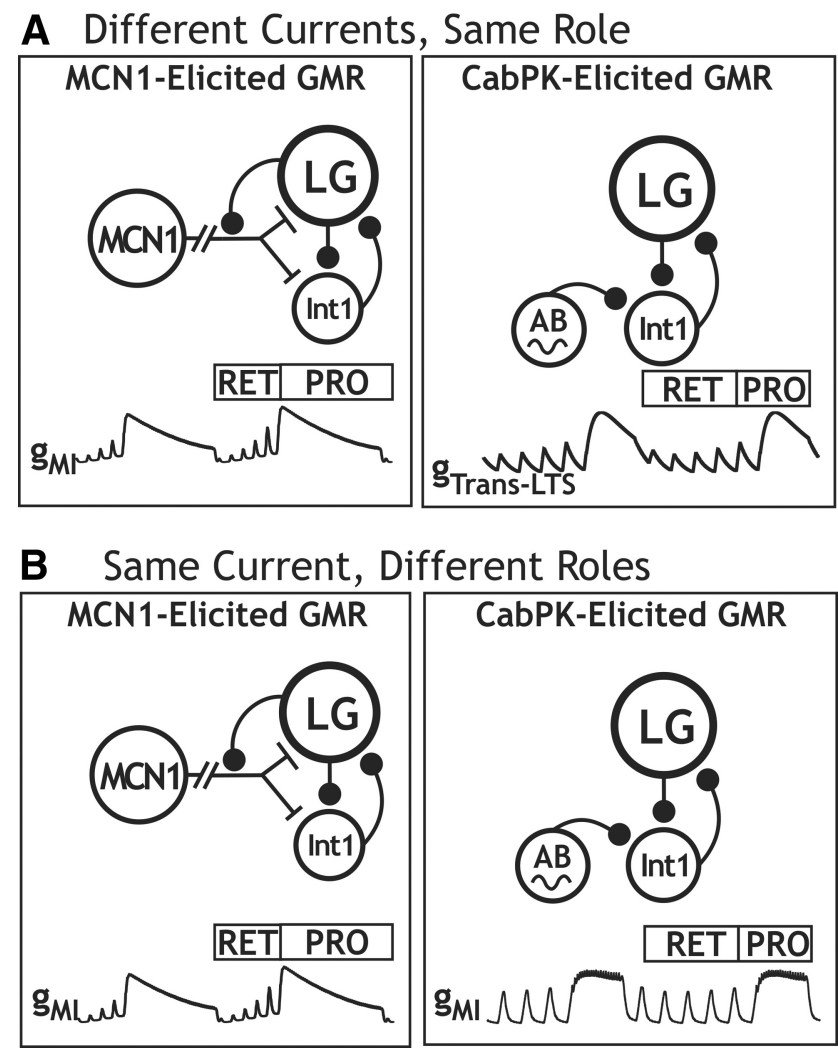

Figure 14. MCN1 stimulation and CabPK superfusion activate different conductances to perform the same function, and activate the same conductance to perform different functions, during gastric mill rhythm generation. $\boldsymbol{A}$, Rhythmic $L G$ neuron burst generation results from the rhythmic buildup and decay of MCN1-activated $g_{\mathrm{MI}}$ (left) and CabPKactivated $g_{\text {Trans-LTS }}$ (right). During MCN1 stimulation, the buildup occurs during retraction and results from continual MCN1 activation of $g_{\mathrm{MI}}$, while the decay during protraction results from $L G$ neuron presynaptic inhibition of MCN1 transmitter release. During CabPK application, the buildup during retraction results from the accumulation of deinactivation in $g_{\text {Trans-LTS }}$, while the decay during protraction results from the time-dependent inactivation of this conductance. $\boldsymbol{B}$, The $g_{\mathrm{MI}}$ in $\mathrm{LG}$ is the burst-generating conductance during the MCN1 - gastric mill rhythm, whereas it provides a sustained but subthreshold depolarizing drive that facilitates PIR burst generation by $I_{\text {Trans-LTS }}$ during the CabPK- gastric mill rhythm. Note that the different $g_{\mathrm{MI}}$ trajectories during protraction result from $g_{\mathrm{MI}}$ being both voltage- and synaptic inhibition-dependent during the MCN1- gastric mill rhythm, whereas during the CabPK - gastric mill rhythm it is only voltage-dependent. RET, Retraction; PRO, protraction.

MCN1 and CabPK activation of the gastric mill rhythm generator involves both divergent actions of the same ionic current $\left(I_{\mathrm{MI}}\right)$ and a conserved function mediated by distinct ionic currents $\left(I_{\mathrm{MI}}, I_{\text {Trans-LTS }} ;\right.$ Fig. 14). As discussed above, $I_{\mathrm{MI}}$ is the burstgenerating conductance during MCN1 rhythm generation, whereas during CabPK rhythm generation there is insufficient $I_{\mathrm{MI}}$ to directly enable LG neuron bursting. In this latter condition, $I_{\mathrm{MI}}$ instead facilitates burst generation by $I_{\text {Trans-LTS }}$. The shared role of $I_{\mathrm{MI}}$ and $I_{\text {Trans-LTS }}$ during MCN1 and CabPK rhythm generation, respectively, results from each current exhibiting a phase-dependent growth and decay process that is pivotal to determining the LG neuron burst (protraction) and interburst (retraction) durations (Fig. 14; Bartos et al., 1999; DeLong et al., 2009a; this article). The growth and decay process for $I_{\mathrm{MI}}$ during $\mathrm{MCN} 1$ stimulation results from its synaptic regulation, as discussed above. In contrast, the availability of CabPK-activated $I_{\text {Trans-LTS }}$ grows and decays during gastric mill retraction and protraction, respectively, due to its voltage- and time-dependent 
properties. During retraction, when the LG neuron is hyperpolarized by rhythmic synaptic inhibition from Int1, $I_{\text {Trans-LTS }}$ exhibits a buildup of deinactivation, which increases its availability, while during protraction it first enables and then limits the LG neuron burst duration due to its depolarization- and timedependent inactivation.

Despite these differences in the rhythm-generating process, during both gastric mill rhythms the LG neuron burst initiates during a pyloric rhythm-timed depolarization that results from AB neuron inhibition of Int1 (Bartos et al., 1999; Saideman et al., 2007b). However, these pyloric-timed depolarizations are necessary only for CabPK rhythm generation (Saideman et al., 2007b). This is because, when the pyloric rhythm is suppressed during MCN1 stimulation, the MCN1activated $I_{\mathrm{MI}}$ amplitude continues to grow during each prolonged retraction phase until it eventually becomes large enough to enable LG to escape from Int 1 inhibition and generate a burst (Bartos et al., 1999). In contrast, CabPK-activated $I_{\mathrm{MI}}$ alone is insufficient to enable the LG neuron to depolarize and trigger $I_{\text {Trans-LTS }}$ activation in the presence of persistent Int 1 inhibition.

The phase transitions occurring during rhythmic alternating bursting by reciprocally inhibitory neurons commonly result from the inhibited neuron either escaping from continuing inhibition ("escape" mode) or waiting until it is released from that inhibition ("release" mode) (Skinner et al., 1994; Marder and Calabrese, 1996; McCormick and Bal, 1997). Each mode can also mediate the same transition under different conditions (Bartos et al., 1999; Sorensen et al., 2004; Kristan et al., 2005). During the MCN1-gastric mill and Cab$\mathrm{PK}$-gastric mill rhythms, the protraction-to-retraction transition appears to occur via the release mechanism, as the LG neuron burst self-terminates and releases Int1 from inhibition. In contrast, for reasons discussed above, during the retraction-to-protraction transition the CabPK rhythm only operates via the release mode, whereas the MCN1 rhythm can operate either in its normal release mode or, if the pyloric rhythm is very slow or suppressed, in the escape mode.

A neural network might have the ability to configure distinct circuits generating the same activity pattern because it needs to generate the same core behavior under different conditions. These differently configured circuits, however, might be differentially sensitive to particular inputs. This, for example, is evident for the pyloric pacemaker neuron $A B$ influence on these two gastric mill circuits (i.e., it is a necessary rhythmgenerating component only for the CabPK-gastric mill circuit). Such differential sensitivity likely also occurs in circuits that generate a consistent output pattern despite extensive, albeit compensatory, changes in the baseline intrinsic and synaptic conductances of circuit neurons (Prinz et al., 2004a; Grashow et al., 2009, 2010; Calabrese et al., 2011; Guttierez et al., 2013). Two interesting tests of this hypothesis for the gastric mill circuit will be the influence of the gastro-pyloric receptor neuron, a muscle proprioceptor, and the peptide hormone CCAP on the CabPK-gastric mill rhythm. Both of these inputs regulate the MCN1-gastric mill rhythm via actions that involve $\mathrm{MCN}$, which does not participate in the CabPK rhythm (Beenhakker et al., 2005; DeLong et al., 2009a,b).

This study highlights an alternative consequence to the well established flexibility in neural circuit output imparted by their neuromodulatory inputs. Here, neuromodulation configures distinct circuits/mechanisms that generate a conserved output rather than generating distinct outputs. As part of this process, different modulatory pathways activate the same ionic current in the same neuron but use it in different ways, and they use different currents in the same neuron to perform the same function (Fig. 14). Whether the conserved output pattern resulting from these distinct mechanisms belies latent differences in responsiveness to the same perturbation remains to be determined.

\section{References}

Angstadt JD, Grassmann JL, Theriault KM, Levasseur SM (2005) Mechanisms of postinhibitory rebound and its modulation by serotonin in excitatory swim motor neurons of the medicinal leech. J Comp Physiol A Neuroethol Sens Neural Behav Physiol 191:715-732. CrossRef Medline

Bal T, Nagy F, Moulins M (1994) Muscarinic modulation of a patterngenerating network: control of neuronal properties. J Neurosci 14:30193035. Medline

Bartos M, Manor Y, Nadim F, Marder E, Nusbaum MP (1999) Coordination of fast and slow rhythmic neuronal circuits. J Neurosci 19:66506660. Medline

Beenhakker MP, Nusbaum MP (2004) Mechanosensory activation of a motor circuit by coactivation of two projection neurons. J Neurosci 24:67416750. CrossRef Medline

Beenhakker MP, DeLong ND, Saideman SR, Nadim F, Nusbaum MP (2005) Proprioceptor regulation of motor circuit activity by presynaptic inhibition of a modulatory projection neuron. J Neurosci 25:8794-8806. CrossRef Medline

Blitz DM, Nusbaum MP (2012) Modulation of circuit feedback specifies motor circuit output. J Neurosci 32:9182-9193. CrossRef Medline

Blitz DM, Christie AE, Coleman MJ, Norris BJ, Marder E, Nusbaum MP (1999) Different proctolin neurons elicit distinct motor patterns from a multifunctional neuronal network. J Neurosci 19:5449-5463. Medline

Blitz DM, Beenhakker MP, Nusbaum MP (2004) Different sensory systems share projection neurons but elicit distinct motor patterns. J Neurosci 24:11381-11390. CrossRef Medline

Briggman KL, Kristan WB (2008) Multifunctional pattern-generating circuits. Annu Rev Neurosci 31:271-294. CrossRef Medline

Calabrese RL, Norris BJ, Wenning A, Wright TM (2011) Coping with variability in small neuronal networks. Integr Comp Biol 51:845-855. CrossRef Medline

Christie AE, Stein W, Quinlan JE, Beenhakker MP, Marder E, Nusbaum MP (2004) Actions of a histaminergic/peptidergic projection neuron on rhythmic motor patterns in the stomatogastric nervous system of the crab Cancer borealis. J Comp Neurol 469:153-169. CrossRef Medline

Coleman MJ, Nusbaum MP (1994) Functional consequences of compartmentalization of synaptic input. J Neurosci 14:6544-6552. Medline

Coleman MJ, Meyrand P, Nusbaum MP (1995) Presynaptic inhibition mediates a switch between two modes of synaptic transmission. Nature 378: 502-505. CrossRef Medline

DeLong ND, Nusbaum MP (2010) Hormonal modulation of sensorimotor integration. J Neurosci 30:2418-2427. CrossRef Medline

DeLong ND, Kirby MS, Blitz DM, Nusbaum MP (2009a) Parallel regulation of a modulator-activated current via distinct dynamics underlies comodulation of motor circuit output. J Neurosci 29:12355-12367. CrossRef Medline

DeLong ND, Beenhakker MP, Nusbaum MP (2009b) Presynaptic inhibition selectively weakens peptidergic cotransmission in a small motor system. J Neurophysiol 102:3492-3504. CrossRef Medline

Derjean D, Moussaddy A, Atallah E, St-Pierre M, Auclair F, Chang S, Ren X, Zielinski B, Dubuc R (2010) A novel neural substrate for the transformation of olfactory inputs into motor output. PLoS Biol 8:e1000567. CrossRef Medline

Dickinson PS (2006) Neuromodulation of central pattern generators in invertebrates and vertebrates. Curr Opin Neurobiol 16:604-614. CrossRef Medline 
Diehl F, White RS, Stein W, Nusbaum MP (2013) Motor circuit-specific burst patterns drive different muscle and movement patterns. J Neurosci 33:12013-12029. CrossRef Medline

Di Prisco GV, Pearlstein E, Le Ray D, Robitaille R, Dubuc R (2000) A cellular mechanism for the transformation of a sensory input into a motor command. J Neurosci 20:8169-8176. Medline

Doi A, Ramirez JM (2008) Neuromodulation and the orchestration of the respiratory rhythm. Respir Physiol Neurobiol 164:96-104. CrossRef Medline

Doi A, Ramirez JM (2010) State-dependent interactions between excitatory neuromodulators in the neuronal control of breathing. J Neurosci 30: 8251-8262. CrossRef Medline

Engbers JD, Anderson D, Tadayonnejad R, Mehaffey WH, Molineux ML, Turner RW (2011) Distinct roles for $\mathrm{I}(\mathrm{T})$ and $\mathrm{I}(\mathrm{H})$ in controlling the frequency and timing of rebound spike responses. J Physiol 589:53915413. CrossRef Medline

Felix RA 2nd, Fridberger A, Leijon S, Berrebi AS, Magnusson AK (2011) Sound rhythms are encoded by postinhibitory rebound spiking in the superior paraolivary nucleus. J Neurosci 31:12566-12578. CrossRef Medline

Goaillard JM, Taylor AL, Schulz DJ, Marder E (2009) Functional consequences of animal-to-animal variation in circuit parameters. Nat Neurosci 12:1424-1430. CrossRef Medline

Golowasch J, Marder E (1992) Proctolin activates an inward current whose voltage dependence is modified by extracellular $\mathrm{Ca}^{2+}$. J Neurosci 12:810817. Medline

Grashow R, Brookings T, Marder E (2009) Reliable neuromodulation from circuits with variable underlying structure. Proc Natl Acad Sci U S A 106: 11742-11746. CrossRef Medline

Grashow R, Brookings T, Marder E (2010) Compensation for variable intrinsic neuronal excitability by circuit-synaptic interactions. J Neurosci 30:9145-9156. CrossRef Medline

Guttierez GJ, O'Leary T, Marder E (2013) Multiple mechanisms switch an electrically coupled, synaptically inhibited neuron between competing rhythmic oscillators. Neuron 77:845-858. CrossRef Medline

Harris-Warrick RM (2011) Neuromodulation and flexibility in central pattern generator networks. Curr Opin Neurobiol 21:685-692. CrossRef Medline

Harris-Warrick RM, Coniglio LM, Levini RM, Gueron S, Guckenheimer J (1995) Dopamine modulation of two subthreshold currents produces phase shifts in activity of an identified motoneuron. J Neurophysiol 74: 1404-1420. Medline

Kadiri LR, Kwan AC, Webb WW, Harris-Warrick RM (2011) Dopamineinduced oscillations of the pyloric pacemaker neuron rely on release of calcium from intracellular stores. J Neurophysiol 106:1288-1298. CrossRef Medline

Kintos N, Nusbaum MP, Nadim F (2008) A modeling comparison of projection neuron- and neuromodulator-elicited oscillations in a central pattern generating network. J Comput Neurosci 24:374-397. CrossRef Medline

Kirby MS, Nusbaum MP (2007) Peptide hormone modulation of a neuronally modulated motor circuit. J Neurophysiol 98:3206-3220. CrossRef Medline

Korn H, Faber DS (2005) The Mauthner cell half a century later: a neurobiological model for decision-making? Neuron 47:13-28. CrossRef Medline

Kristan WB Jr, Calabrese RL, Friesen WO (2005) Neuronal control of leech behavior. Prog Neurobiol 76:279-327. CrossRef Medline

MacLean JN, Zhang Y, Johnson BR, Harris-Warrick RM (2003) Activityindependent homeostasis in rhythmically active neurons. Neuron 37: 109-120. CrossRef Medline

Marder E (2012) Neuromodulation of neuronal circuits: back to the future. Neuron 76:1-11. CrossRef Medline

Marder E, Bucher D (2007) Understanding circuit dynamics using the stomatogastric nervous system of lobsters and crabs. Annu Rev Physiol 69: 291-316. CrossRef Medline

Marder E, Calabrese RL (1996) Principles of rhythmic motor pattern generation. Physiol Rev 76:687-717. Medline

Marder E, Eisen JS (1984) Transmitter identification of pyloric neurons: electrically coupled neurons use different transmitters. J Neurophysiol 51:1345-1361. Medline
McCormick DA, Bal T (1997) Sleep and arousal: thalamocortical mechanisms. Annu Rev Neurosci 20:185-215. CrossRef Medline

Nadim F, Manor Y, Nusbaum MP, Marder E (1998) Frequency regulation of a slow rhythm by a fast periodic input. J Neurosci 18:5053-5067. Medline

Norris BJ, Coleman MJ, Nusbaum MP (1994) Recruitment of a projection neuron determines gastric mill motor pattern selection in the stomatogastric nervous system of the crab, Cancer borealis. J Neurophysiol 72: 1451-1463. Medline

Norris BJ, Wenning A, Wright TM, Calabrese RL (2011) Constancy and variability in the output of a central pattern generator. J Neurosci 31: 4663-4674. CrossRef Medline

Nusbaum MP, Marder E (1989a) A modulatory proctolin-containing neuron (MPN). I. Identification and characterization. J Neurosci 9:15911599. Medline

Nusbaum MP, Marder E (1989b) A modulatory proctolin-containing neuron (MPN). II. State-dependent modulation of rhythmic motor activity. J Neurosci 9:1600-1607. Medline

Pirtle TJ, Willingham K, Satterlie RA (2010) A hyperpolarization-activated inward current alters swim frequency of the pteropod mollusk Clione limacina. Comp Biochem Physiol A Mol Integr Physiol 157:319-327. CrossRef Medline

Prinz AA, Bucher D, Marder E (2004a) Similar network activity from disparate circuit parameters. Nat Neurosci 7:1345-1352. CrossRef Medline

Prinz AA, Abbott LF, Marder E (2004b) The dynamic clamp comes of age. Trends Neurosci 27:218-224. CrossRef Medline

Ransdell JL, Temporal S, West NL, Leyrer ML, Schulz DJ (2013) Characterization of inward currents and channels underlying burst activity in motoneurons of crab cardiac ganglion. J Neurophysiol 110:42-54. CrossRef Medline

Rauscent A, Einum J, Le Ray D, Simmers J, Combes D (2009) Opposing aminergic modulation of distinct spinal locomotor circuits and their functional coupling during amphibian metamorphosis. J Neurosci 29: 1163-1174. CrossRef Medline

Robinson RB, Siegelbaum SA (2003) Hyperpolarization-activated cation currents: from molecules to physiological function. Annu Rev Physiol 65: 453-480. CrossRef Medline

Saideman SR, Ma M, Kutz-Naber KK, Cook A, Torfs P, Schoofs L, Li L, Nusbaum MP (2007a) Modulation of rhythmic motor activity by pyrokinin peptides. J Neurophysiol 97:579-595. CrossRef Medline

Saideman SR, Blitz DM, Nusbaum MP (2007b) Convergent motor patterns from divergent circuits. J Neurosci 27:6664-6674. CrossRef Medline

Sangrey T, Jaeger D (2010) Analysis of distinct short and prolonged components in rebound spiking of deep cerebellar nucleus neurons. Eur J Neurosci 32:1646-1657. CrossRef Medline

Sekirnjak C, du Lac S (2002) Intrinsic firing dynamics of vestibular nucleus neurons. J Neurosci 22:2083-2095. Medline

Sharp AA, O'Neil MB, Abbott LF, Marder E (1993) The dynamic clamp: artificial conductances in biological neurons. Trends Neurosci 16:389394. CrossRef Medline

Skinner FK, Kopell N, Marder E (1994) Mechanisms for oscillation and frequency control in reciprocally inhibitory model neural networks. J Comput Neurosci 1:69-87. CrossRef Medline

Sorensen M, DeWeerth S, Cymbalyuk G, Calabrese RL (2004) Using a hybrid neural system to reveal regulation of neuronal network activity by an intrinsic current. J Neurosci 24:5427-5438. CrossRef Medlineb

Stein W (2009) Modulation of stomatogastric rhythms. J Comp Physiol A Neuroethol Sens Neural Behav Physiol 195:989-1009. CrossRef Medline

Stein W, DeLong ND, Wood DE, Nusbaum MP (2007) Divergent cotransmitter actions underlie motor pattern activation by a modulatory projection neuron. Eur J Neurosci 26:1148-1165. CrossRef Medline

Swensen AM, Marder E (2000) Multiple peptides converge to activate the same voltage-dependent current in a central pattern-generating circuit. J Neurosci 20:6752-6759. Medline

Swensen AM, Marder E (2001) Modulators with convergent cellular actions elicit distinct circuit outputs. J Neurosci 21:4050-4058. Medline

Turrigiano GG, Marder E, Abbott LF (1996) Cellular short-term memory from a slow potassium conductance. J Neurophysiol 75:963-966. Medline 
Wang D, Grillner S, Wallén P (2011) 5-HT and dopamine modulates CaV1.3 calcium channels involved in postinhibitory rebound in the spinal network for locomotion in lamprey. J Neurophysiol 105:1212-1224. CrossRef Medline

Weimann JM, Meyrand P, Marder E (1991) Neurons that form multiple pattern generators: identification and multiple activity patterns of gastric/ pyloric neurons in the crab stomatogastric system. J Neurophysiol 65: 111-122. Medline

White RS, Nusbaum MP (2011) The same core rhythm generator underlies different rhythmic motor patterns. J Neurosci 31:11484-11494. CrossRef Medline

Wood DE, Stein W, Nusbaum MP (2000) Projection neurons with shared cotransmitters elicit different motor patterns from the same neural circuit. J Neurosci 20:8943-8953. Medline

Wood DE, Manor Y, Nadim F, Nusbaum MP (2004) Intercircuit control via rhythmic regulation of projection neuron activity. J Neurosci 24:74557463. CrossRef Medline

Zhang B, Wootton JF, Harris-Warrick RM (1995) Calcium-dependent plateau potentials in a crab stomatogastric ganglion motor neuron. II. Calciumactivated slow inward current. J Neurophysiol 74:1938-1946. Medline

Zheng N, Raman IM (2011) Prolonged postinhibitory rebound firing in the cerebellar nuclei mediated by group I metabotropic glutamate receptor potentiation of L-type calcium currents. J Neurosci 31:10283-10292. CrossRef Medline 